\title{
A double copy for asymptotic symmetries in the self-dual sector
}

\section{Miguel Campiglia ${ }^{a}$ and Silvia Nagy ${ }^{b}$}

${ }^{a}$ Facultad de Ciencias, Universidad de la República, Igúa 4225, Montevideo, Uruguay

${ }^{b}$ Centre for Research in String Theory, School of Physics and Astronomy, Queen Mary University of London, 327 Mile End Road, London E1 4NS, U.K.

E-mail: campi@fisica.edu.uy, s.nagy@qmul.ac.uk

ABSTRACT: We give a double copy construction for the symmetries of the self-dual sectors of Yang-Mills (YM) and gravity, in the light-cone formulation. We find an infinite set of double copy constructible symmetries. We focus on two families which correspond to the residual diffeomorphisms on the gravitational side. For the first one, we find novel non-perturbative double copy rules in the bulk. The second family has a more striking structure, as a non-perturbative gravitational symmetry is obtained from a perturbatively defined symmetry on the YM side.

At null infinity, we find the YM origin of the subset of extended Bondi-Metzner-Sachs (BMS) symmetries that preserve the self-duality condition. In particular, holomorphic large gauge YM symmetries are double copied to holomorphic supertranslations. We also identify the single copy of superrotations with certain non-gauge YM transformations that to our knowledge have not been previously presented in the literature.

Keywords: Gauge Symmetry, Space-Time Symmetries, Classical Theories of Gravity, Integrable Field Theories

ArXiv EPrint: 2102.01680 


\section{Contents}

1 Introduction 1

2 Self-dual fields $\quad 3$

2.1 Symmetries of the self-dual field equations 5

3 Residual gauge symmetries 5

3.1 YM field 6

$\begin{array}{lll}3.2 & \text { Metric field } & 7\end{array}$

$\begin{array}{lll}3.2 .1 & \text { First family of symmetries } & 7\end{array}$

$\begin{array}{ll}3.2 .2 & \text { Second family of symmetries }\end{array}$

4 Perturbative approach to symmetries $\quad 10$

4.1 YM field 10

4.2 Metric field 11

4.2.1 First family of symmetries $\quad 11$

$\begin{array}{ll}4.2 .2 \text { Second family of symmetries } & 12\end{array}$

5 Color to kinematics map for symmetries $\quad 12$

$\begin{array}{lll}5.1 & \text { First family of symmetries } & 12\end{array}$

$\begin{array}{ll}5.2 \text { Second family of symmetries } & 13\end{array}$

$\begin{array}{lll}5.2 .1 \mathcal{O}\left(\Phi^{1}\right) \text { symmetry } & 14\end{array}$

$\begin{array}{lll}5.2 .2 \mathcal{O}\left(\phi^{1}\right) \text { symmetry from the DC } & 15\end{array}$

$\begin{array}{lll}5.3 \text { Summary } & 15\end{array}$

$\begin{array}{ll}\text { 5.4 Relation with the symmetry raising map } & 16\end{array}$

$\begin{array}{ll}5.5 & \text { Double copy for an infinite family of symmetries } \\ \end{array}$

$6 \quad$ Large gauge symmetries at null infinity $\quad 19$

$\begin{array}{lll}6.1 & \text { Asymptotic fields at null infinity } & 19\end{array}$

$\begin{array}{lll}6.2 & \text { Review of large gauge symmetries at null infinity } 20\end{array}$

$\begin{array}{lll}6.3 & \text { Residual gauge symmetries at null infinity } & 21\end{array}$

7 Color to kinematic symmetry map at null infinity 23

$\begin{array}{lll}7.1 & \text { First family } 23\end{array}$

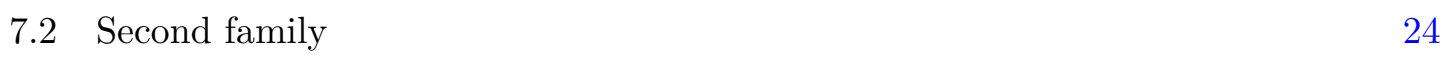

$\begin{array}{lll}7.2 .1 & \tilde{\delta} \text { from } \Lambda=O\left(r^{0}\right), \lambda=O(r) & 25\end{array}$

7.2.2 $\tilde{\delta}$ from $\Lambda=O(r), \lambda=O\left(r^{2}\right) \quad 26$

$\begin{array}{lll}7.3 & \text { Summary } & 28\end{array}$

$\begin{array}{llr}8 & \text { Discussion } & 29\end{array}$ 
A.1 Yang-Mills field 30

A.2 Metric field 31

B Perturbative transformations at higher orders $\quad 32$

B.1 Second order 32

B.1.1 First family 32

B.1.2 Second family 33

B.2 Recursive construction at arbitrary orders 35

C Comparison with convolution DC $\quad 36$

\section{Introduction}

The idea of gravity as a double copy (DC $)^{1}$ has found applications in the study of numerous aspects of the gravitational theory, most notably scattering amplitudes, where much of the recent success has been driven by the identification of a duality between the color and kinematic factors, the so-called Bern-Carrasco-Johannson (BCJ) duality [4-11]. It has progressed to the study of solutions, both perturbative [12-18] and exact [19-35]. Another important extension has been to find manifestations of it beyond gravity theory [36-42]. A natural line of work following from these successes has been to formalise the correspondence by constructing double copy dictionaries for the fields themselves and by studying the symmetries of the theories, both local [43-48] and global [49-53], as well as by giving direct constructions of Lagrangians via the DC [6, 54-59].

Having a DC prescription for the local symmetries of the fields (i.e. the gauge transformations on the Yang-Mills (YM) side and diffeomorphisms on the gravity side) is of course a proof of the robustness of the DC dictionaries for the fields, but the possible applications of such a prescription go beyond this. For example, it was shown in [40,44, 58], working in the BRST formalism, that one can exploit it to obtain a gauge-mapping algorithm, which gives a gravity gauge fixing functional output from a YM gauge-fixing functional input. This has been extended to the study of classical solutions in [16]. However, this has so far only been understood at linear level - going beyond this, either perturbatively (or nonperturbatively in certain special cases), could prove very powerful in the DC construction of classical solutions and/or Lagrangians.

A natural arena in which to discuss gravitational and gauge symmetries is the asymptotic boundary of spacetime. In the case of (asymptotically) flat spacetimes, this is given by Penrose's null infinity [60]. It has long been known that gauge and gravitational fields exhibit a rich structure at this boundary, with one of the earliest examples being the BondiMetzner-Sachs (BMS) asymptotic symmetry group of gravity [61, 62]. Whereas many of the implications of these symmetries were known since the 80's thanks to Ashtekar and

\footnotetext{
${ }^{1}$ See $[1-3]$ for some comprehensive reviews.
} 
others [63-65], the subject experienced a veritable renaissance in recent years. The trigger was twofold. On the one hand, Barnich and Troessaert proposed an extension of BMS to include so-called superrotations [66]. On the other hand, Strominger discovered a link between future and past null infinity symmetries [67] which manifests as soft theorems in scattering amplitudes $[68,69]$. The ramifications of these developments are still ongoing, see $[70-72]$ for reviews.

One may then ask whether the asymptotic perspective could shed light into the DC (and viceversa). In fact, there has already been progress in this direction, through the use of DC for soft theorems [73-80] and in the context of celestial amplitudes [81-84]. Additionally, a duality transformation relating the Schwarzschild and Taub-NUT solutions was shown to arise, via a DC construction, from an electric-magnetic duality relation $[85,86]$. Interestingly, this duality can be interpreted, on the gravity side, as the complexification of a particular supertranslation transformation. ${ }^{2}$ These promising results are calling for an interpretation from the perspective of asymptotic symmetries.

In this paper we present some progress in both directions outlined above. We will be restricting to the self-dual sector of gravity and YM theory, where the colour-kinematics duality was made manifest at the level of the algebra by Monteiro and O'Connell [87] (see also [11, 38, 88-92] for related work and extensions), leading to a simple DC prescription for the construction of perturbative solutions. In light-cone gauge, the self-dual graviton and YM field have a natural description in terms of scalar fields $\phi$ and $\Phi$, respectively [93-101], and the self-duality conditions can be expressed as equations for these scalars, referred to as self-dual Einstein (SDE) and self-dual YM (SDYM) equations respectively. We will study the symmetries of these equations, with focus on those arising from the residual gauge symmetries of their respective parent theories. After laying down the 'bulk' DC symmetry map (see below for details), we will move on to study symmetries at null infinity. On the gravitational side we will recover the subset of BMS that preserves the self-duality condition at null infinity. On the YM side, the single copy version of these symmetries will turn out to include large gauge as well as certain non-gauge symmetries. The latter, to our knowledge, have not been discussed before in the asymptotic symmetry literature.

The SDYM and SDE equations are known to possess an infinite ladder of symmetries [94, 95, 99, 100, 102-106], out of which the symmetries described above turn out to represent only two rungs. We will see that the DC applies to all levels of this ladder. The asymptotic analysis of the higher level symmetries could in principle be worked out along the same lines as the ones studied here, but we do not attempt to do so in this work.

Let us now describe in more detail our proposed DC symmetry map. As anticipated above, we focus on two families of symmetries. The first is exact in both YM and gravity, and constitutes a subset of diffeomorphisms on the gravity side and a subset of the nonabelian gauge symmetry on the YM side. We discover that a repackaging is required in order to manifest the color-kinematic duality and we find the appropriate way to perform the DC. Crucially, we find that the gauge parameter of YM is not mapped to the diffeo-

\footnotetext{
${ }^{2}$ It appears this duality could be the asymptotic version of the Ehlers transform discussed in [46, 47]. It would be interesting to make this connection explicitly. We thank Eoin O Colgain for bringing this possibility to our attention.
} 
morphism parameter of gravity, but to a "Hamiltonian" of the diffeomorphism vector field w.r.t. the Poisson bracket inherent in the self-dual theory. Having performed this repackaging, the DC rules become remarkably simple. The second family is also exact on the gravity side, but perturbative and non-local on the YM side, presenting an apparent puzzle. However, we find that the repackaging described above recasts the gravity transformation as a perturbative non-local series. This is then obtainable from its YM counterpart via the same simple rules as the first family, verifying the robustness of our construction. It is interesting to note that this second family is a subset of diffeomorphisms on the gravity side, but its single copy is not a subset of YM gauge transformations. In the asymptotic limit, the first family maps holomorphic YM large gauge symmetries to holomorphic supertranslations, whereas the second one identifies certain (non-gauge and non-local) SDYM transformations to holomorphic superrotations. ${ }^{3}$

The paper is structured as follows: in section 2 we introduce our conventions and present a covariant description of self-dual fields in light-cone gauge. The corresponding residual gauge symmetries are discussed in section 3. In section 4 we describe a perturbative approach to general symmetries of the SDYM and SDE equations. In section 5 we construct the DC map between the symmetries of these equations. Starting with a non-perturbative map for the first family of symmetries, we eventually extend its applicability to an infinite number of families. In section 6 we introduce coordinates adapted to null infinity and review gauge and gravity asymptotic symmetries. In section 7 we take the null-infinity limit of the first two families of symmetries described earlier and establish the DC map for asymptotic symmetries. We summarize our findings and discuss possible future avenues in section 8. Some of the technical discussions are given in appendices.

\section{Self-dual fields}

Consider Minkowski spacetime written in light-cone coordinates $(U, V, Z, \bar{Z})$, related to Cartesian coordinates $X^{\mu}$ by:

$$
U=\frac{X^{0}-X^{3}}{\sqrt{2}}, \quad V=\frac{X^{0}+X^{3}}{\sqrt{2}}, \quad Z=\frac{X^{1}+i X^{2}}{\sqrt{2}}, \quad \bar{Z}=\frac{X^{1}-i X^{2}}{\sqrt{2}} .
$$

We will split these light-cone coordinates in the following two sets of $2 d$ coordinates:

$$
x^{i}:=(U, \bar{Z}), \quad y^{\alpha}:=(V, Z) .
$$

The spacetime metric then takes the form

$$
d s^{2}=2 \eta_{i \alpha} d x^{i} d y^{\alpha}=-2 d U d V+2 d Z d \bar{Z} .
$$

The description of self-dual fields and their symmetries will involve the "area element" of these $2 \mathrm{~d}$ spaces, described by

$$
\begin{aligned}
\Omega_{i j} d x^{i} d x^{j} & =d U d \bar{Z}-d \bar{Z} d U \\
\Pi_{\alpha \beta} d y^{\alpha} d y^{\beta} & =d V d Z-d Z d V
\end{aligned}
$$

\footnotetext{
${ }^{3}$ We have here simplified the discussion in the interest of clarity. Asymptotically, the second family splits into two subfamilies, see figure 1 for the complete picture.
} 
We will treat $\Omega_{i j}$ and $\Pi_{\alpha \beta}$ as antisymmetric 4 dimensional tensors with $\Omega_{\alpha \mu}$ and $\Pi_{i \mu}$ vanishing. By raising indices with the inverse spacetime metric $\eta^{i \alpha}$, we can regard these two tensors as (partial) inverses of each other:

$$
\begin{aligned}
\Omega_{i}{ }^{\alpha} \Pi_{\alpha}^{j} & =\delta_{i}^{j} \\
\Pi_{\alpha}{ }^{i} \Omega_{i}{ }^{\beta} & =\delta_{\alpha}^{\beta} .
\end{aligned}
$$

The self-dual YM and metric fields will be described in terms of scalar fields $\Phi$ and $\phi$ according to

$$
\begin{aligned}
\mathcal{A}_{\mu} & =\Pi_{\mu}^{\nu} \partial_{\nu} \Phi \\
h_{\mu \nu} & =\Pi_{\mu}{ }^{\rho} \Pi_{\nu}{ }^{\sigma} \partial_{\rho} \partial_{\sigma} \phi .
\end{aligned}
$$

In the notation of (2.2), the non-zero components take the form

$$
\begin{aligned}
\mathcal{A}_{\alpha} & =\Pi_{\alpha}^{i} \partial_{i} \Phi \\
h_{\alpha \beta} & =\Pi_{\alpha}^{i} \Pi_{\beta}^{j} \partial_{i} \partial_{j} \phi,
\end{aligned}
$$

with the scalar fields satisfying ${ }^{4}$

$$
\begin{aligned}
& \square \Phi=-i \Pi^{i j}\left[\partial_{i} \Phi, \partial_{j} \Phi\right] \\
& \square \phi=\frac{1}{2} \Pi^{i j} \Pi^{k l} \partial_{i} \partial_{k} \phi \partial_{j} \partial_{l} \phi
\end{aligned}
$$

where $\square \equiv 2 \eta^{i \alpha} \partial_{i} \partial_{\alpha}$ is the wave operator. We will refer to (2.12) and (2.13) as the self-dual YM (SDYM) and self-dual Einstein (SDE) equation respectively. We are using conventions where the coupling constants are absorbed in the field, so that the field strength is $\mathcal{F}_{\mu \nu}=\partial_{\mu} \mathcal{A}_{\nu}-\partial_{\nu} \mathcal{A}_{\mu}-i\left[\mathcal{A}_{\mu}, \mathcal{A}_{\mu}\right]$, the spacetime metric $g_{\mu \nu}=\eta_{\mu \nu}+h_{\mu \nu}$, and there is no explicit coupling constant in the field equations.

In [87], the notion of a kinematic algebra in the self-dual sector was defined as a Poisson bracket algebra. In our conventions, the Poisson bracket $(\mathrm{PB})$ is defined as:

$$
\{f, g\}:=\Pi^{i j} \partial_{i} f \partial_{j} g
$$

One can now recover (2.13) from (2.12) via the replacement rules [87]:

$$
-i[,] \rightarrow \frac{1}{2}\{,\} . \quad \Phi \rightarrow \phi .
$$

Note that our conventions are slightly different from [87], and so are the color to kinematics replacement rules.

\footnotetext{
${ }^{4}$ See appendix A for the derivation of these equations from the self-duality conditions for YM and gravity.
} 


\subsection{Symmetries of the self-dual field equations}

The self-dual YM and Einstein equations have long been known to possess infinitely many symmetries [94, 95, 99, 100, 102-104]. These can be constructed recursively as follows. Let $\delta \Phi$ and $\delta \phi$ be symmetries of the SDYM and SDE equations respectively:

$$
\begin{aligned}
\square \delta \Phi & =-2 i \Pi^{i j}\left[\partial_{i} \Phi, \partial_{j} \delta \Phi\right] \\
\square \delta \phi & =\Pi^{i j} \Pi^{k l} \partial_{i} \partial_{k} \phi \partial_{j} \partial_{l} \delta \phi
\end{aligned}
$$

One can then obtain new symmetries $\tilde{\delta} \Phi$ and $\tilde{\delta} \phi$, defined implicitly by the condition ${ }^{5}$

$$
\begin{aligned}
\partial_{i} \tilde{\delta} \Phi & =\Omega_{i}{ }^{\alpha} \partial_{\alpha} \delta \Phi-i\left[\partial_{i} \Phi, \delta \Phi\right] \\
\partial_{i} \tilde{\delta} \phi & =\Omega_{i}{ }^{\alpha} \partial_{\alpha} \delta \phi+\frac{1}{2}\left\{\partial_{i} \phi, \delta \phi\right\}
\end{aligned}
$$

In each case, one can verify the consistency condition $\partial_{[i} \partial_{j} \tilde{\delta}=0$ is satisfied. By direct computation one can then check that $\tilde{\delta} \Phi$ and $\tilde{\delta} \phi$ satisfy (2.16) and (2.17) provided the respective SDYM and SDE equations are satisfied. We will refer to this $\delta \rightarrow \tilde{\delta}$ map as the symmetry raising map on each respective theory. It will turn out to be useful for identifying the DC rules for asymptotic symmetries.

We conclude the section by noting that the scalar fields also have intrinsic redundancies: the field $\Phi$ should be thought of as defined modulo an arbitrary function $\Psi(y)$ :

$$
\Phi \sim \Phi+\Psi(y)
$$

as this does not affect the YM field (2.10). Similarly, $\phi$ is defined modulo

$$
\phi \sim \phi+\psi^{(0)}(y)+x^{i} \psi_{i}^{(1)}(y) .
$$

which preserves (2.11). ${ }^{6}$ Note that (2.20) and (2.21) are not the standard gauge symmetries of the YM and metric fields (to be discussed in the next section). Rather, they are additional redundancies that arise in the description given by (2.10) and (2.11).

\section{$3 \quad$ Residual gauge symmetries}

In this section we describe the gauge transformations on the YM and gravity fields that preserve the form (2.10), (2.11). It is interesting to note that these fields satisfy the following gauge conditions

$$
\partial^{\mu} \mathcal{A}_{\mu}=0, \quad \partial^{\mu} h_{\mu \nu}=0, \quad \eta^{\mu \nu} h_{\mu \nu}=0 .
$$

Thus, the symmetries that we will be discussing can be thought of as a subset of the residual gauge symmetries of conditions (3.1).

\footnotetext{
${ }^{5}$ See e.g. eq. (15) of [98] for the YM case.

${ }^{6}$ The attentive reader might notice that whereas (2.20) is a symmetry of the field equation (2.12), this does not appear to be the case for (2.21) and (2.13). The reason for this is that the actual self-dual condition on the metric, which is of course invariant under (2.21), takes the form of a differential operator acting on (2.13) - see section A.2.
} 


\subsection{YM field}

We start with the YM field. Given a gauge parameter $\Lambda$, the corresponding gauge transformation is

$$
\delta_{\Lambda} \mathcal{A}_{\mu}=\partial_{\mu} \Lambda+i\left[\Lambda, \mathcal{A}_{\mu}\right]
$$

If we want to preserve the condition $\mathcal{A}_{i}=0$, we must have

$$
\partial_{i} \Lambda=0 \Longrightarrow \Lambda=\Lambda(y)
$$

We now ask if a gauge transformation with such gauge parameter is compatible with (2.10). That is, we ask whether there is a $\delta_{\Lambda} \Phi$ such that

$$
\delta_{\Lambda} \mathcal{A}_{\alpha}=\Pi_{\alpha}^{i} \partial_{i} \delta_{\Lambda} \Phi
$$

By substituting (2.10) in (3.2), the l.h.s. of (3.4) can be written as

$$
\begin{aligned}
\delta_{\Lambda} \mathcal{A}_{\alpha} & =\partial_{\alpha} \Lambda+i\left[\Lambda, \Pi_{\alpha}{ }^{i} \partial_{i} \Phi\right] \\
& =\partial_{\alpha} \Lambda+i \Pi_{\alpha}{ }^{i} \partial_{i}[\Lambda, \Phi],
\end{aligned}
$$

where to get the last line we used (3.3). The second term in (3.6) is of the required form (3.4). We find it convenient to use the notation

$$
\delta_{\Lambda} \Phi=\delta_{\Lambda}^{(0)} \Phi+\delta_{\Lambda}^{(1)} \Phi
$$

where $\delta_{\Lambda}^{(0)} \Phi$ is independent of $\Phi$ and $\delta_{\Lambda}^{(1)} \Phi$ is linear in $\Phi$. We emphasize that this is an exact symmetry and that we are not doing a perturbative expansion at this stage. Eq. (3.6) then tells us that

$$
\delta_{\Lambda}^{(1)} \Phi=i[\Lambda, \Phi]
$$

It now remains to see whether the first term in (3.6) can also be written in the form (3.4). That is, we would like to find $\delta_{\Lambda}^{(0)} \Phi$ such that

$$
\Pi_{\alpha}^{i} \partial_{i} \delta_{\Lambda}^{(0)} \Phi=\partial_{\alpha} \Lambda
$$

Using (2.6), this equation can equivalently be written as:

$$
\partial_{i} \delta_{\Lambda}^{(0)} \Phi=\Omega_{i}{ }^{\alpha} \partial_{\alpha} \Lambda
$$

Since the r.h.s. is independent of $x^{i}$, we can integrate it directly to obtain ${ }^{7}$

$$
\delta_{\Lambda}^{(0)} \Phi=\Omega_{i}^{\alpha} x^{i} \partial_{\alpha} \Lambda
$$

Summarizing: the gauge symmetries compatible with (2.10) are parametrized by gauge parameters $\Lambda(y)$, and act on $\Phi$ according to

$$
\delta_{\Lambda} \Phi=\Omega_{i}{ }^{\alpha} x^{i} \partial_{\alpha} \Lambda+i[\Lambda, \Phi] .
$$

Additionally, one could also consider symmetries of the scalar $\Phi$ which do not arise as YM gauge transformations, but are intrinsic symmetries of the equation (2.12). As we will show in section 5 , one of these families of transformations can be obtained as the single copy of a gravitational diffeomorphism symmetry.

\footnotetext{
${ }^{7}$ We ignore an integration 'constant' $\Psi(y)$ due to the redundancy $(2.20)$.
} 


\subsection{Metric field}

In the gravitational case, the spacetime metric is given by

$$
g_{\mu \nu}=\eta_{\mu \nu}+h_{\mu \nu}
$$

Let us emphasize this is not just a first order metric but the full-non linear (self-dual) metric. In particular, the inverse metric is exactly given by $g^{\mu \nu}=\eta^{\mu \nu}-h^{\mu \nu}$ since $\eta^{\rho \sigma} h_{\mu \rho} h_{\sigma \nu}=0$. Except for the metric tensor $g$, we will be raising and lowering indices with $\eta$.

Given a vector field $\xi^{\mu}$, the gauge transformation on $h_{\mu \nu}$ is defined from the lie derivative on $g_{\mu \nu}$ :

$$
\delta_{\xi} h_{\mu \nu}:=\mathcal{L}_{\xi} \eta_{\mu \nu}+\mathcal{L}_{\xi} h_{\mu \nu}
$$

We will be looking for vector fields that are independent of $h$, so we can study each term in (3.14) separately. We start by imposing the condition that the first term in (3.14) preserves $h_{i \mu}=0$ :

$$
\mathcal{L}_{\xi} \eta_{i \mu}=2 \partial_{(i} \xi_{\mu)}=0
$$

where $\xi_{\mu} \equiv \eta_{\mu \nu} \xi^{\nu}$, with $\xi^{\nu}$ the infinitesimal diffeomorphism vector field. Setting $\mu=i, \alpha$ we obtain

$$
2 \partial_{(i} \xi_{j)}=0 \Longrightarrow \xi_{i}=a_{i j}(y) x^{j}-c_{i}(y) \quad \text { with } a_{(i j)}=0
$$

and

$$
\partial_{i} \xi_{\alpha}+\partial_{\alpha} \xi_{i}=0 \Longrightarrow \partial_{i} \xi_{\alpha}=-\partial_{\alpha} a_{i j} x^{j}+\partial_{\alpha} c_{i}
$$

An equation of the form $\partial_{i} \xi_{\alpha}=v_{i \alpha}$ can be solved only if $\partial_{[i} v_{j] \alpha}=0$. This implies

$$
\partial_{\alpha} a_{i j}=0 .
$$

A constant $a_{i j}$ represents a rigid rotation in the $x^{i}$ plane and is hence part of the global Poincare symmetries. Since we are interested in local symmetries, we set $a_{i j}=0$. Integrating (3.17) we are led to

$$
\xi_{\alpha}=\partial_{\alpha} c_{i}(y) x^{i}+b_{\alpha}(y)
$$

with $b_{\alpha}(y)$ an integration "constant". To continue, we need to (i) determine if the second term in (3.14) is compatible with $h_{i \mu}=0$ and (2.11) and (ii) determine if $2 \partial_{(\alpha} \xi_{\beta)}$ can be written in a form compatible with (2.11). To simplify the analysis, we will discuss separately the cases $c_{i}=0$ and $b_{\alpha}=0$. The general case can be obtained as a linear combination of these two simple cases.

\subsubsection{First family of symmetries}

Setting $c_{i}=0$, we have

$$
\xi_{i}=0, \quad \xi_{\alpha}=b_{\alpha}(y) .
$$

and so the resulting vector field is given $b^{8}$

$$
\xi^{i}=\eta^{i \alpha} b_{\alpha}(y), \quad \xi^{\alpha}=0 .
$$

\footnotetext{
${ }^{8}$ Recall we raise and lower indices with $\eta$. For the vector field (3.21) this happens to be equivalent to raising and lowering with $g$, but this will not be always the case.
} 
Let us now discuss the second term in (3.14)

$$
\mathcal{L}_{\xi} h_{\mu \nu}=\xi^{\rho} \partial_{\rho} h_{\mu \nu}+2 \partial_{(\mu} \xi^{\rho} h_{\nu) \rho} .
$$

From (3.21) and $h_{i \mu}=0$ we find that the second term in (3.22) vanishes. The first term clearly respects $h_{i \mu}=0$. Furthermore, using eq. (2.11) it can be written as

$$
\begin{aligned}
\xi^{k} \partial_{k} h_{\alpha \beta} & =\xi^{k} \partial_{k} \Pi_{\alpha}^{i} \Pi_{\beta}^{j} \partial_{i} \partial_{j} \phi \\
& =\Pi_{\alpha}^{i} \Pi_{\beta}^{j} \partial_{i} \partial_{j}\left(\xi^{k} \partial_{k} \phi\right)
\end{aligned}
$$

where in the second line we used the fact that $\partial_{i} \xi^{k}=0$. Eq. (3.24) gives the part of $\delta_{\xi} \phi$ that is linear in $\phi$ :

$$
\delta_{\xi}^{(1)} \phi=\xi^{k} \partial_{k} \phi
$$

It remains to see whether the first term in (3.14) is compatible with (2.11). Thus, we ask if there is a $\delta_{\xi}^{(0)} \phi$ such that

$$
\Pi_{\alpha}^{i} \Pi_{\beta}^{j} \partial_{i} \partial_{j} \delta_{\xi}^{(0)} \phi=2 \partial_{(\alpha} \xi_{\beta)} .
$$

Using (2.6) and (3.20) this equation can be written as:

$$
\partial_{i} \partial_{j} \delta_{\xi}^{(0)} \phi=2 \Omega_{i}{ }^{\alpha} \Omega_{j}^{\beta} \partial_{(\alpha} b_{\beta)} .
$$

Since $b_{\beta}$ is independent of the $x^{i}$ variables, the equation can be directly integrated, leading to $^{9}$

$$
\delta_{\xi}^{(0)} \phi=\Omega_{i}{ }^{\alpha} \Omega_{j}{ }^{\beta} x^{i} x^{j} \partial_{\alpha} b_{\beta} .
$$

The family of vector fields (3.21) then acts on the scalar field according to

$$
\delta_{\xi} \phi=\Omega_{i}{ }^{\alpha} \Omega_{j}{ }^{\beta} x^{i} x^{j} \partial_{\alpha} b_{\beta}+\eta^{i \alpha} b_{\alpha} \partial_{i} \phi .
$$

At this stage we notice that $\delta_{\xi} \phi$ does not appear to be a symmetry of the SDE equation (2.13). To see what has gone wrong, we need to recall (see appendix A) that the SDE equation for $\phi(2.13)$ is a sufficient but not necessary condition to produce a self-dual metric. The actual self-duality condition takes the form of a 2 nd order differential operator acting on the SDE equation (2.13), see eqs. (A.21) and (A.23). Of course the self-duality condition is invariant under the transformation (3.29), since it arises from an infinitesimal diffeomorphism. In order to have a symmetry of the SDE equation (2.13), one can check that it is sufficient to restrict $b_{\alpha}(y)$ to be a total derivative,

$$
b_{\alpha}(y)=\partial_{\alpha} b(y)
$$

We will refer to the resulting symmetry transformation as $\delta_{b}$, with:

$$
\delta_{b} \phi=\Omega_{i}{ }^{\alpha} \Omega_{j}{ }^{\beta} x^{i} x^{j} \partial_{\alpha} \partial_{\beta} b+\eta^{i \alpha} \partial_{\alpha} b \partial_{i} \phi .
$$

We will later see that (3.30) does not actually represent any restriction on the asymptotic vector fields at null infinity.

\footnotetext{
${ }^{9}$ As in the YM case, we do not include the 'integration constants' that are part of the ambiguity in the definition of $\phi(2.21)$.
} 


\subsubsection{Second family of symmetries}

Setting $b_{\alpha}=0$ we have

$$
\xi_{i}=-c_{i}(y), \quad \xi_{\alpha}=\partial_{\alpha} c_{j}(y) x^{j} .
$$

We now start by demanding the first term in (3.14) to be compatible with (2.11), as this will impose restrictions on $c_{i}$ :

$$
2 \partial_{(\alpha} \xi_{\beta)}=2 \partial_{\alpha} \partial_{\beta} c_{k}(y) x^{k}=\Pi_{\alpha}^{i} \Pi_{\beta}^{j} \partial_{i} \partial_{j} \delta_{\xi}^{(0)} \phi
$$

for some $\delta_{\xi}^{(0)} \phi$. Multiplying the equation with $\Omega$ 's as before, the last equality is equivalent to

$$
\partial_{i} \partial_{j} \delta_{\xi}^{(0)} \phi=2 \Omega_{i}{ }^{\alpha} \Omega_{j}{ }^{\beta} \partial_{\alpha} \partial_{\beta} c_{k}(y) x^{k}=: c_{i j k} x^{k} .
$$

For this to be integrable, we need the tensor $c_{i j k}$ to be symmetric under the exchange of any two indices. The expression as given is already symmetric in $(i j)$. One can show that, in order to make it symmetric in $(j k)$ (and hence in $(i k)$ ) one needs to take

$$
c_{k}(y)=\Omega_{k}^{\gamma} \partial_{\gamma} c(y)
$$

for some function $c(y)$. The resulting expression can then be integrated, leading to

$$
\delta_{\xi}^{(0)} \phi=\frac{1}{3} \Omega_{i}{ }^{\alpha} \Omega_{j}{ }^{\beta} \Omega_{k}^{\gamma} x^{i} x^{j} x^{k} \partial_{\alpha} \partial_{\beta} \partial_{\gamma} c .
$$

We finally discuss the second term in (3.14) for the vector field $\xi^{\mu}$ :

$$
\xi^{i}=\eta^{i \alpha} \Omega_{j}{ }^{\beta} x^{j} \partial_{\alpha} \partial_{\beta} c, \quad \xi^{\alpha}=-\Omega^{\alpha \beta} \partial_{\beta} c .
$$

One can show that such a vector field preserves the condition $h_{i \mu}=0$ as well as the form (2.11). To simplest way to show this is to verify the vector field leaves the tensor $\Pi_{\mu}{ }^{\nu}$ invariant

$$
\mathcal{L}_{\xi} \Pi_{\mu}{ }^{\nu}=0,
$$

from which it follows that $\mathcal{L}_{\xi} h_{i \mu}=0$ and

$$
\mathcal{L}_{\xi} h_{\alpha \beta}=\frac{1}{4} \Pi_{\alpha}^{i} \Pi_{\beta}^{j} \partial_{i} \partial_{j} \delta_{\xi}^{(1)} \phi
$$

where

$$
\delta_{\xi}^{(1)} \phi=\mathcal{L}_{\xi} \phi=\xi^{i} \partial_{i} \phi+\xi^{\alpha} \partial_{\alpha} \phi
$$

To summarize, the second family of vector fields acts on $\phi$ as: $\delta_{\xi} \phi=\delta_{\xi}^{(0)} \phi+\delta_{\xi}^{(1)} \phi$, with $\delta_{\xi}^{(0)} \phi$ and $\delta_{\xi}^{(1)} \phi$ given by (3.36) and (3.40) respectively.

We emphasize that both families are exact, non-perturbative symmetries of the field equations (2.13). As we will discuss in section 5.4, it turns out that the second family can be obtained from the first one by the symmetry raising map given in eq. (2.19). 


\section{Perturbative approach to symmetries}

In this section we present a perturbative approach to symmetries of the SDYM and SDE equations. This will offer an alternative perspective on the symmetries described before. The perturbative approach will be useful when constructing the DC symmetry map in section 5. We will present expressions up to first order in the fields. The all-order expansions are given recursively in section B.2.

\subsection{YM field}

The field equations (2.12) may be cast as a tower of field equations associated to a perturbative expansion ${ }^{10}$

$$
\Phi=\Phi^{(0)}+\Phi^{(1)}+\cdots
$$

such that

$$
\begin{aligned}
\square \Phi^{(0)} & =0 \\
\square \Phi^{(1)} & =-i \Pi^{i j}\left[\partial_{i} \Phi^{(0)}, \partial_{j} \Phi^{(0)}\right] \\
\ldots &
\end{aligned}
$$

We now look at symmetries $\delta \Phi$ of the field equations (2.12) in the above perturbative scheme. That is, we look for

$$
\delta \Phi=\delta \Phi^{(0)}+\delta \Phi^{(1)}+\cdots
$$

such that

$$
\begin{aligned}
\square \delta \Phi^{(0)} & =0 \\
\square \delta \Phi^{(1)} & =-2 i \Pi^{i j}\left[\partial_{i} \Phi^{(0)}, \partial_{j} \delta \Phi^{(0)}\right] \\
\ldots &
\end{aligned}
$$

We may think of these equations as determining $\delta \Phi$ in terms of a "seed" $\delta \Phi^{(0)}$ : given $\delta \Phi^{(0)}$ satisfying (4.6), $\delta \Phi^{(1)}$ is defined implicitly by (4.7), and similarly for the higher order terms. Generically this would lead to a $\delta \Phi^{(1)}$ that depends non-locally on $\Phi^{(0)}$ due to the need to invert the wave operator. The case of gauge symmetries discussed previously is an exception. From (3.11) we see that in this case the seed is given by

$$
\delta \Phi^{(0)}=\Omega_{j}{ }^{\beta} x^{j} \partial_{\beta} \Lambda(y),
$$

which satisfies (4.6):

$$
\square \delta \Phi^{(0)}=2 \eta^{i \alpha} \partial_{i} \partial_{\alpha} \delta \Phi^{(0)}=2 \Omega^{\alpha \beta} \partial_{\alpha} \partial_{\beta} \Lambda=0 .
$$

\footnotetext{
${ }^{10}$ Recall we have absorbed the coupling constant in the definition of the field; the order in the expansion counts the number of $\Phi^{(0)}$ fields.
} 
If we now substitute (4.9) in the r.h.s. of (4.7) we find

$$
\begin{aligned}
-2 i \Pi^{i j}\left[\partial_{i} \Phi^{(0)}, \partial_{j} \delta \Phi^{(0)}\right] & =-2 i \eta^{i \beta}\left[\partial_{i} \Phi^{(0)}, \partial_{\beta} \Lambda\right] \\
& =-i \square\left[\Phi^{(0)}, \Lambda\right]
\end{aligned}
$$

where in the first equality we used $\Pi^{i j} \Omega_{j}{ }^{\beta}=\eta^{i \beta}$ and in the second equality we used (4.2) and the fact that $\Lambda$ is independent of $x^{i}$. From (4.7) we then conclude that

$$
\delta \Phi^{(1)}=-i\left[\Phi^{(0)}, \Lambda\right],
$$

consistent with the result from the previous section. One could continue and obtain the higher order terms, which in this case will resum to the exact symmetry (3.12). See appendix B for further discussion on higher order terms.

\subsection{Metric field}

Proceeding analogously for the "metric" field $\phi$, we have

$$
\begin{aligned}
\phi & =\phi^{(0)}+\phi^{(1)}+\cdots, \\
\square \phi^{(0)} & =0 \\
\square \phi^{(1)} & =\frac{1}{2} \Pi^{i j} \Pi^{k l} \partial_{i} \partial_{k} \phi^{(0)} \partial_{j} \partial_{l} \phi^{(0)} \\
\ldots &
\end{aligned}
$$

A symmetry $\delta \phi=\delta \phi^{(0)}+\delta \phi^{(1)}+\cdots$ of the field equations will then satisfy

$$
\begin{aligned}
\square \delta \phi^{(0)} & =0 \\
\square \delta \phi^{(1)} & =\Pi^{i j} \Pi^{k l} \partial_{i} \partial_{k} \phi^{(0)} \partial_{j} \partial_{l} \delta \phi^{(0)} \\
\ldots &
\end{aligned}
$$

We now discuss how the residual gauge symmetries found in the previous section fit into this description.

\subsubsection{First family of symmetries}

For the first family of vector fields described earlier, eq. (3.31) gives the following candidate for the "seed" $\delta \phi^{(0)}$ :

$$
\delta \phi^{(0)}=\Omega_{i}{ }^{\alpha} \Omega_{j}{ }^{\beta} x^{i} x^{j} \partial_{\alpha} \partial_{\beta} b,
$$

which satisfies $\square \delta \phi^{(0)}=0$. If we now substitute (4.21) in the r.h.s. of (4.19) we obtain

$$
\begin{aligned}
\Pi^{i j} \Pi^{k l} \partial_{i} \partial_{k} \phi^{(0)} \partial_{j} \partial_{l} \delta \phi^{(0)} & =2 \eta^{k \beta} \eta^{i \alpha} \partial_{i} \partial_{k} \phi^{(0)} \partial_{\alpha} \partial_{\beta} b \\
& =\square \eta^{i \alpha} \partial_{i} \phi^{(0)} \partial_{\alpha} b
\end{aligned}
$$

where to get the last equality we used (4.18) and the fact that $b$ is independent of $x^{i}$. Comparing with (4.19) we conclude

$$
\delta \phi^{(1)}=\eta^{i \alpha} \partial_{i} \phi^{(0)} \partial_{\alpha} b
$$

compatible with the expected result from (3.31). 


\subsubsection{Second family of symmetries}

For the second family of symmetries, we take $\delta \phi^{(0)}$ to be given by (3.36)

$$
\delta \phi^{(0)}=\frac{1}{3} \Omega_{i}{ }^{\alpha} \Omega_{j}{ }^{\beta} \Omega_{k}^{\gamma} x^{i} x^{j} x^{k} \partial_{\alpha} \partial_{\beta} \partial_{\gamma} c,
$$

It can be easily checked that the above satisfies (4.18). Substituting in the r.h.s. of (4.19) one finds

$$
\Pi^{i j} \Pi^{k l} \partial_{i} \partial_{k} \phi^{(0)} \partial_{j} \partial_{l} \delta \phi^{(0)}=-2 \eta^{i \beta} \eta^{l \gamma} \Omega_{m}^{\alpha} x^{m} \partial_{i} \partial_{l} \phi^{(0)} \partial_{\alpha} \partial_{\beta} \partial_{\gamma} c .
$$

Since it is not immediately obvious how to "pull out" a d'Alambertian from such an expression, let us take guidance from the previous section. From (3.40) and (3.37) we expect to have

$$
\delta \phi^{(1)}=\eta^{i \alpha} \Omega_{j}{ }^{\beta} x^{j} \partial_{i} \phi^{(0)} \partial_{\alpha} \partial_{\beta} c-\Omega^{\alpha \beta} \partial_{\alpha} \phi^{(0)} \partial_{\beta} c .
$$

Indeed, one can check by direct computation that the d'Alambertian of (4.27) coincides with (4.26), upon using (4.15).

\section{Color to kinematics map for symmetries}

According to [87], the color to kinematics map for the self-dual sector consists in replacing YM matrix commutators by certain Poisson brackets on the $x^{i}$ variables, as reviewed in $(2.15)$ :

$$
\Phi \rightarrow \phi, \quad-i[,] \rightarrow \frac{1}{2}\{,\}
$$

This will be our starting point in understanding the double copy for symmetries. In the following section, we will make the color-kinematics duality manifest at the level of the symmetry transformations. A key ingredient in this will be to recast the gravitational symmetry in a form that is analogous to the YM one. We will achieve this by expressing the gravitational symmetry parameter in terms of a Hamiltonian $\lambda$ (w.r.t. the Poisson bracket) associated to the diffeomorphism vector field. This will allow us to supplement the rules (5.1) with

$$
\Lambda \rightarrow \lambda
$$

where $\Lambda$ is the YM symmetry parameter and $\lambda$ is the Hamiltonian describing the gravitational symmetry.

\subsection{First family of symmetries}

We will start by comparing the gauge symmetries found in the YM case (3.12) with the first family of symmetries found in the gravitational case (3.31):

$$
\begin{aligned}
\delta_{\Lambda} \Phi & =\Omega_{i}{ }^{\alpha} x^{i} \partial_{\alpha} \Lambda+i[\Lambda, \Phi] \\
\delta_{b} \phi & =\Omega_{i}{ }^{\alpha} \Omega_{j}{ }^{\beta} x^{i} x^{j} \partial_{\alpha} \partial_{\beta} b+\eta^{i \alpha} \partial_{\alpha} b \partial_{i} \phi .
\end{aligned}
$$

Let us first focus on the terms that are linear in the scalar fields. According to (5.1), the YM term linear in $\Phi$ should be mapped to

$$
-i[\Lambda, \Phi] \rightarrow \frac{1}{2}\{\lambda, \phi\}
$$


for some $\lambda$. In order to reproduce the linear term in $\delta_{b} \phi$ we take

$$
\lambda=2 \Omega_{i}{ }^{\alpha} x^{i} \partial_{\alpha} b
$$

which can be thought of as the "Hamiltonian" of the vector field $\xi^{i}=\eta^{i \alpha} \partial_{\alpha} b$ with respect to the Poisson bracket $\frac{1}{2}\{$,$\} .$

We now notice that the $\phi$-independent piece of $\delta_{b} \phi$ can be written in terms of $\lambda$, leading to an expression that has the same form as the $\Phi$-independent piece of $\delta_{\Lambda} \Phi$. Thus, we can express the total $\delta_{b} \phi$ in terms of $\lambda$ as:

$$
\delta_{b} \phi=\frac{1}{2} \Omega_{i}{ }^{\alpha} x^{i} \partial_{\alpha} \lambda-\frac{1}{2}\{\lambda, \phi\} .
$$

Comparing the expression above with (5.3), we find that the tranformation rules map into each other under

$$
\Phi \rightarrow \phi, \quad-i[,] \rightarrow \frac{1}{2}\{,\}, \quad \Lambda \rightarrow \lambda
$$

with an apparent mismatch of $\frac{1}{2}$ for the inhomogeneous terms. However we note that this is resolved by expressing the transformation rules on the YM and gravitational fields themselves, as:

$$
\begin{aligned}
\delta_{\Lambda} \mathcal{A}_{\alpha} & =\partial_{\alpha} \Lambda+i \Pi_{\alpha}{ }^{i} \partial_{i}[\Lambda, \Phi] \\
\delta_{\lambda} h_{\alpha \beta} & =\Pi_{(\alpha}^{i} \partial_{i}\left(\partial_{\beta)} \lambda-\frac{1}{2} \Pi_{\beta)}^{j} \partial_{j}\{\lambda, \phi\}\right)
\end{aligned}
$$

If we insist on working at the level of the scalar fields, then the replacement rules need to be augmented by the inclusion of a multiplicative factor for the inhomogeneous term:

$$
\mathfrak{r}=\frac{\operatorname{deg}(\Lambda)+1}{\operatorname{deg}(\lambda)+1}
$$

where $\operatorname{deg}(f)$ counts the power of $x^{i}$ in a function $f$ which is homogeneous in the $x^{i}$ variables. This numerical factor dissapears in the replacement rules for

$$
\mathcal{A}_{\alpha}=\Pi_{\alpha}^{i} \partial_{i} \Phi \rightarrow h_{\alpha \beta}=\Pi_{\alpha}^{i} \Pi_{\beta}^{j} \partial_{i} \partial_{j} \phi
$$

due to the action of the derivatives w.r.t. $x^{i}$ on the inhomogeneous term.

Finally, we note that

$$
\operatorname{deg}(\lambda)=\operatorname{deg}(\Lambda)+1
$$

and moreover

$$
\Lambda(y) \rightarrow \lambda=2 \Omega_{i}{ }^{\alpha} x^{i} \partial_{\alpha} b(y)
$$

\subsection{Second family of symmetries}

Having understood how the first family of gravitational symmetries can be obtained from the YM ones, we now focus on the second family of gravitational symmetries:

$$
\delta_{c} \phi=\frac{1}{3} \Omega_{i}^{\alpha} \Omega_{j}^{\beta} \Omega_{k}^{\gamma} x^{i} x^{j} x^{k} \partial_{\alpha} \partial_{\beta} \partial_{\gamma} c+\xi^{i} \partial_{i} \phi+\xi^{\alpha} \partial_{\alpha} \phi
$$


where

$$
\xi^{i}=\eta^{i \alpha} \Omega_{j}{ }^{\beta} x^{j} \partial_{\alpha} \partial_{\beta} c, \quad \xi^{\alpha}=-\Omega^{\alpha \beta} \partial_{\beta} c .
$$

We first note that the vector field $\xi^{i}$ can again be written in terms of a 'Hamiltonian'

$$
\tilde{\lambda}=\Omega_{i}{ }^{\alpha} \Omega_{j}{ }^{\beta} x^{i} x^{j} \partial_{\alpha} \partial_{\beta} c
$$

so that $\xi^{i} \partial_{i} \phi=\frac{1}{2}\{\phi, \tilde{\lambda}\}$. Using (5.16), we can rewrite (5.14) as

$$
\delta_{c} \phi=\frac{1}{3} \Omega_{i}{ }^{\alpha} x^{i} \partial_{\alpha} \tilde{\lambda}-\frac{1}{2}\{\tilde{\lambda}, \phi\}-\Omega^{\alpha \beta} \partial_{\alpha} \phi \partial_{\beta} c .
$$

This brings $\delta_{c} \phi$ into a form that resembles the one obtained before for $\delta_{b} \phi$ in (5.7), with the exception of the final term. To understand this extra term we will use the perturbative description of symmetries given in section 4 , as described below.

We would like to find a symmetry of the SDYM theory such that, upon the appropriate color to kinematics replacement rules, one recovers the second family of gravitational symmetries (5.17). Guided by (5.13), we find that the natural choice for the $\Phi$-independent piece of this YM symmetry is

$$
\delta_{\tilde{\Lambda}}^{(0)} \Phi=\frac{1}{2} \Omega_{i}{ }^{\alpha} x^{i} \partial_{\alpha} \tilde{\Lambda}
$$

with $\tilde{\Lambda}$ of the form

$$
\tilde{\Lambda}=\Omega_{i}{ }^{\alpha} x^{i} \partial_{\alpha} B(y)
$$

for some function $B(y)$. This generalizes the $\Phi$-independent part of the symmetry (5.3) to the case where $\Lambda$ is linear in $x$. Restricting to the inhomogeneous terms in the transformations for the YM field and the graviton,

$$
\begin{aligned}
\delta_{\tilde{\Lambda}}^{(0)} \mathcal{A}_{\alpha} & =\partial_{\alpha} \tilde{\Lambda} \\
\delta_{\tilde{\lambda}}^{(0)} h_{\alpha \beta} & =\Pi_{(\alpha}^{i} \partial_{i} \partial_{\beta)} \tilde{\lambda}
\end{aligned}
$$

we find that they are related by the same rules (5.8) as the first family. ${ }^{11}$ Alternatively, one can work directly at the level of the scalar fields by taking into acount the multiplicative factor (5.10), which in this case is $\mathfrak{r}=2 / 3$, using $\operatorname{deg}(\tilde{\Lambda})=1$ and $\operatorname{deg}(\tilde{\lambda})=2$.

The idea now is to use the perturbative method of section 4 to obtain the $\mathcal{O}\left(\Phi^{1}\right)$ symmetry transformation generated by (5.18). We will then discuss how the color to kinematic rules applied to this term reproduce the two $\mathcal{O}\left(\phi^{1}\right)$ terms in (5.17).

\subsection{1 $\mathcal{O}\left(\Phi^{1}\right)$ symmetry}

Following the perturbative method of section 4 , we would like to solve for $\delta_{\tilde{\Lambda}} \Phi^{(1)}$ in eq. (4.7),

$$
\square \delta_{\tilde{\Lambda}} \Phi^{(1)}=-2 i \Pi^{i j}\left[\partial_{i} \Phi^{(0)}, \partial_{j} \delta_{\tilde{\Lambda}} \Phi^{(0)}\right]
$$

\footnotetext{
${ }^{11}$ We emphasize here that $\delta_{\tilde{\Lambda}}$ is not defined directly on $\mathcal{A}_{\mu}$, but through its action on the scalar $\Phi$. In particular, using (2.8), we find $\delta_{\tilde{\Lambda}}^{(0)} \mathcal{A}_{i} \equiv \Pi_{i}{ }^{\mu} \partial_{\mu} \delta_{\tilde{\Lambda}}^{(0)} \Phi=0$, due the fact that $\Pi_{i}{ }^{\mu}$ vanishes identically. Note that $\delta_{\tilde{\Lambda}}^{(0)} \mathcal{A}_{i} \neq \partial_{i} \tilde{\Lambda}$, so it cannot be interpreted as a gauge transformation, even at the lowest order.
} 
given the 'seed'

$$
\delta_{\tilde{\Lambda}} \Phi^{(0)}=\frac{1}{2} \Omega_{i}{ }^{\alpha} x^{i} \partial_{\alpha} \tilde{\Lambda}
$$

We proceed in a similar fashion as in the analysis between eqs. (4.9) and (4.12) where we verified the perturbative method against the residual YM gauge symmetry. Noting that $\partial_{j} \delta_{\tilde{\Lambda}} \Phi^{(0)}=\Omega_{j}{ }^{\alpha} \partial_{\alpha} \tilde{\Lambda}$, the r.h.s. of (5.21) can be written

$$
\begin{aligned}
-2 i \Pi^{i j}\left[\partial_{i} \Phi^{(0)}, \partial_{j} \delta_{\tilde{\Lambda}} \Phi^{(0)}\right] & =-2 i \eta^{i \alpha}\left[\partial_{i} \Phi^{(0)}, \partial_{\alpha} \tilde{\Lambda}\right] \\
& =-i \square\left[\Phi^{(0)}, \tilde{\Lambda}\right]+2 i \eta^{i \alpha}\left[\partial_{\alpha} \Phi^{(0)}, \partial_{i} \tilde{\Lambda}\right]
\end{aligned}
$$

where in the last line we used that $\square \Phi^{(0)}=0=\square \tilde{\Lambda}$. Unlike for the residual YM symmetry, there is now a reminder term when one 'pulls out' the wave operator. We then conclude that

$$
\delta_{\tilde{\Lambda}} \Phi^{(1)}=-i\left[\Phi^{(0)}, \tilde{\Lambda}\right]+2 i \square^{-1} \eta^{i \alpha}\left[\partial_{\alpha} \Phi^{(0)}, \partial_{i} \tilde{\Lambda}\right] .
$$

\subsection{2 $\mathcal{O}\left(\phi^{1}\right)$ symmetry from the DC}

If we apply the color to kinematic map

$$
-i[,] \rightarrow \frac{1}{2}\{,\}, \quad \Phi \rightarrow \phi, \quad \tilde{\Lambda} \rightarrow \tilde{\lambda}
$$

to expression (5.25) one finds

$$
\delta_{\tilde{\Lambda}} \Phi^{(1)} \rightarrow \frac{1}{2}\left\{\phi^{(0)}, \tilde{\lambda}\right\}-\square^{-1} \eta^{i \alpha}\left\{\partial_{\alpha} \phi^{(0)}, \partial_{i} \tilde{\lambda}\right\}
$$

The first term corresponds to the second term in (5.17). For the second term we have

$$
\begin{aligned}
-\square^{-1} \eta^{i \alpha}\left\{\partial_{\alpha} \phi^{(0)}, \partial_{i} \tilde{\lambda}\right\} & =-\square^{-1} \eta^{i \alpha} \Pi^{j k} \partial_{j} \partial_{\alpha} \phi^{(0)} \partial_{k} \partial_{i} \tilde{\lambda} \\
& =-2 \square^{-1} \eta^{i \alpha} \Pi^{j k} \partial_{j} \partial_{\alpha} \phi^{(0)} \Omega_{i}{ }^{\beta} \Omega_{k}^{\gamma} \partial_{\beta} \partial_{\gamma} c \\
& =-2 \square^{-1} \Omega^{\alpha \beta} \eta^{j \gamma} \partial_{j} \partial_{\alpha} \phi^{(0)} \partial_{\beta} \partial_{\gamma} c \\
& =-2 \square^{-1} \Omega^{\alpha \beta} \eta^{j \gamma} \partial_{j} \partial_{\gamma}\left(\partial_{\alpha} \phi^{(0)} \partial_{\beta} c\right) \\
& =-\Omega^{\alpha \beta} \partial_{\alpha} \phi^{(0)} \partial_{\beta} c,
\end{aligned}
$$

where we used the definition of the Poisson bracket, the expression for $\tilde{\lambda}$ as given in (5.16), and the fact that $\square \phi^{(0)}$ vanishes. Expression (5.32) reproduces, in the perturbative setting, the last term in (5.17). This establishes $\delta_{\tilde{\Lambda}} \Phi^{(1)}$ as the inverse DC map of $\delta_{c} \phi^{(1)}$. In fact, as shown in appendix B the DC map can be extended to arbitrary order in the perturbative expansion.

\subsection{Summary}

Let us summarize our findings. By associating certain "Hamiltonians" to the infinitesimal residual diffeomorphisms of the SDE equations, we managed to obtain simple DC rules mapping SDYM to SDE symmetries. In order to compactly write these rules for the scalar fields, let us introduce the notation

$$
S:=\Omega_{i}{ }^{\alpha} x^{i} \partial_{\alpha} .
$$


The first family of symmetries take the form

$$
\begin{aligned}
\delta_{\Lambda} \Phi & =S(\Lambda)+i[\Lambda, \Phi] \\
\delta_{\lambda} \phi & =\frac{1}{2} S(\lambda)-\frac{1}{2}\{\lambda, \phi\},
\end{aligned}
$$

where on the YM side we have a gauge transformation with parameter $\Lambda$ independent of $x^{i}$, and on the gravitational side we have an infinitesimal diffeomorphism with 'Hamiltonian' $\lambda$ that is linear in $x^{i}$. The two are related by the DC map (5.8), supplemented by the multiplicative coefficient $\mathfrak{r}$ in the inhomogeneous term (see discussion around (5.10) for details):

$$
\Phi \rightarrow \phi, \quad-i[,] \rightarrow \frac{1}{2}\{,\}, \quad \Lambda \rightarrow \lambda, \quad S \rightarrow \mathfrak{r} S, \quad \mathfrak{r}=\frac{\operatorname{deg}(\Lambda)+1}{\operatorname{deg}(\lambda)+1}
$$

The second family can be written as

$$
\begin{aligned}
\delta_{\tilde{\Lambda}} \Phi & =\frac{1}{2} S(\tilde{\Lambda})-i[\Phi, \tilde{\Lambda}]+2 i \square^{-1} \eta^{i \alpha}\left[\partial_{\alpha} \Phi, \partial_{i} \tilde{\Lambda}\right]+\mathcal{O}\left(\Phi^{2}\right) \\
\delta_{\tilde{\lambda}} \phi & =\frac{1}{3} S(\tilde{\lambda})+\frac{1}{2}\{\phi, \tilde{\lambda}\}-\square^{-1} \eta^{i \alpha}\left\{\partial_{\alpha} \phi, \partial_{i} \tilde{\lambda}\right\}+\mathcal{O}\left(\phi^{2}\right)
\end{aligned}
$$

The two are related by exactly the same DC rules (5.36) as for the first family. Although here we have presented the argument up to linear order in the field, the map can in fact be extended to arbitrary order, see appendix B. Let us emphasize that the original gravitational symmetry, as given in (5.17), is non-perturbative. In (5.38), the higher order terms arise as a result of parametrizing this symmetry in terms of the Hamiltonian $\tilde{\lambda}$. This requires the use of the field equations, which introduce higher order terms.

The second family displays two interesting features. Firstly, it maps a perturbative expression on the YM side to a non-perturbative one on the gravity side, as described above. Secondly, while on the gravity side the symmetry is a diffeomorphism, on the YM side we have a non-gauge symmetry of the field equation (2.12).

\subsection{Relation with the symmetry raising map}

In section 2.1 we described the existence of symmetry raising maps on YM and gravity self-dual theories that, given a symmetry $\delta$, produce a new symmetry $\tilde{\delta}$ according to eqs. (2.18), (2.19).

Since, as described in section 4 the symmetries we are studying are fully determined by their inhomogenous piece, it is natural to restrict these equations to the inhomogenous parts of the symmetries:

$$
\begin{aligned}
\partial_{i} \tilde{\delta}^{(0)} \Phi & =\Omega_{i}{ }^{\alpha} \partial_{\alpha} \delta \Phi^{(0)} \\
\partial_{i} \tilde{\delta}^{(0)} \phi & =\Omega_{i}{ }^{\alpha} \partial_{\alpha} \delta \phi^{(0)} .
\end{aligned}
$$

It is now straightforward to check that, if we take $\delta \Phi^{(0)}=\delta_{\Lambda} \Phi^{(0)}$ and $\delta \phi^{(0)}=\delta_{\lambda} \phi^{(0)}$, the resulting $\tilde{\delta}^{(0)}$ are given by $\delta_{\tilde{\Lambda}}^{(0)} \Phi$ and $\delta_{\tilde{\lambda}}^{(0)} \phi$ with

$$
\begin{aligned}
& \tilde{\Lambda}=\delta_{\Lambda}^{(0)} \Phi=S(\Lambda) \\
& \tilde{\lambda}=\delta_{\lambda}^{(0)} \phi=\frac{1}{2} S(\lambda) .
\end{aligned}
$$


Since the symmetries are fully determined by their inhomogenous piece, the above shows that the map $\delta \rightarrow \tilde{\delta}$ takes the full $\delta_{\Lambda} \Phi$ into the full $\delta_{\tilde{\Lambda}} \Phi$ (and similarly for $\delta_{\lambda} \phi$ ). We can summarize the above structure by the diagram

$$
\begin{array}{ccc}
\delta_{\Lambda} \Phi & \stackrel{\mathrm{DC}}{\longrightarrow} & \delta_{\lambda} \phi \\
\downarrow & & \downarrow \\
\delta_{\tilde{\Lambda}} \Phi & \stackrel{\mathrm{DC}}{\longrightarrow} & \delta_{\tilde{\lambda}} \phi,
\end{array}
$$

where the horizontal arrows represent the DC map between each family of symmetries, and the vertical arrows the $\delta \rightarrow \tilde{\delta}$ map on each theory.

Said in different words, we have verified that the symmetry raising map commutes with the DC symmetry map. We will use this result in the next section to obtain the DC symmetry map at null infinity for the second family of symmetries.

\subsection{Double copy for an infinite family of symmetries}

We found that the second family of symmetries arises from applying the raising map on the first family. It is clear that one can continue this process indefinitely and construct an infinite tower of symmetries on both sides. A natural question is whether the DC will continue to hold at each level. In other words, we want to check whether the diagram (5.43) extends indefinitely:

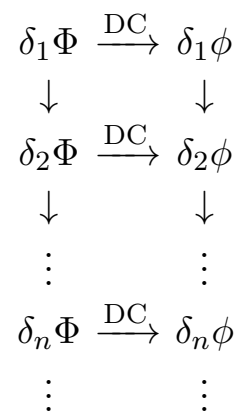

where $\delta_{n}$ denotes the symmetry of the $n$-th family. The first two lines of the diagram correspond to the original diagram (5.43). It is easy to show by recursion that the DC holds at every level. We have explicitly demonstrated this for $\delta_{1}$ and $\delta_{2}$ in section 5.1, section 5.2, and appendix B. Assuming that the DC holds at level $n-1$, it immediately follows from the definition of the $n$-th level symmetry (2.18) and (2.19):

$$
\begin{aligned}
& \partial_{i} \delta_{n} \Phi=\Omega_{i}{ }^{\alpha} \partial_{\alpha} \delta_{n-1} \Phi-i\left[\partial_{i} \Phi, \delta_{n-1} \Phi\right] \\
& \partial_{i} \delta_{n} \phi=\Omega_{i}{ }^{\alpha} \partial_{\alpha} \delta_{n-1} \phi+\frac{1}{2}\left\{\partial_{i} \phi, \delta_{n-1} \phi\right\}
\end{aligned}
$$

that $\delta_{n} \phi$ is obtained from $\delta_{n} \Phi$ via the DC rules (5.36). The focus in this paper is on the first two families, since they are the ones corresponding to asymptotic diffeomorphisms on the gravitational side. It is however instructive to display the general form of the inhomogenous $n$-th transformations. Consider first the YM case, starting with the 'zeroth' $n=0$ family:

$$
\delta_{0} \Phi=\Lambda(y)
$$


Notice that these are just the redundancies described in (2.20). It is then easy to solve (5.45) at $\mathcal{O}\left(\Phi^{0}\right)$. One finds

$$
\delta_{n}^{(0)} \Phi=\frac{1}{n !} S^{n}(\Lambda)
$$

As we did for the second family in (5.41), we can introduce a gauge-like parameter

$$
\Lambda_{n}=\delta_{n-1}^{(0)} \Phi=\frac{1}{(n-1) !} S^{n-1}(\Lambda)
$$

at $n$-th level, such that

$$
\delta_{n}^{(0)} \Phi=\frac{1}{n} S\left(\Lambda_{n}\right)=\frac{1}{\operatorname{deg}\left(\Lambda_{n}\right)+1} S\left(\Lambda_{n}\right)
$$

We refer to $\Lambda_{n}$ as a gauge-like parameter since

$$
\delta_{n}^{(0)} \mathcal{A}_{\alpha}=\Pi_{\alpha}^{i} \partial_{i} \delta_{n}^{(0)} \Phi=\partial_{\alpha} \Lambda_{n}
$$

Note however that $\delta_{n}^{(0)} \mathcal{A}_{i}=0 \neq \partial_{i} \Lambda_{n}$ (see Hfootnote 11 for $n=2$ ). Let us now discuss the gravitational case. Recall that in this case there are two kind of redundancies (2.21). In order to be consistent with the diagram (5.44), one is lead to start the recursion at $n=-1$ with,

$$
\delta_{-1} \phi=2 b(y)
$$

Solving (5.46) at $\mathcal{O}\left(\phi^{0}\right)$. One finds

$$
\delta_{n}^{(0)} \phi=\frac{1}{(n+1) !} S^{n+1}(2 b)=\frac{1}{(n+1) !} S^{n}(\lambda), \quad \lambda:=S(2 b),
$$

where we expressed the result in terms of the first family Hamiltonian $\lambda$. The $n=-1,0$ levels describe the two redundancies in (2.21). As we did for the second family (5.42), we can define a 'Hamiltonian' parameter

$$
\lambda_{n}=\delta_{n-1}^{(0)} \phi=\frac{1}{n !} S^{n-1}(\lambda)
$$

at $n$-th level, such that

$$
\delta_{n}^{(0)} \phi=\frac{1}{n+1} S\left(\lambda_{n}\right)=\frac{1}{\operatorname{deg}\left(\lambda_{n}\right)+1} S\left(\lambda_{n}\right) .
$$

As in the first two families, with this definition of $\lambda_{n}$ one has

$$
\delta_{n}^{(0)} h_{\alpha \beta}=\Pi_{(\alpha}^{i} \partial_{i} \partial_{\beta)} \lambda_{n},
$$

corresponding with the DC version of (5.51). Comparing (5.50) with (5.55) we verify these are indeed compatible with (5.36). The DC map can be extended to arbitrary order in the fields, following section B.2. This extends the applicability of the rules (5.36) to all $n$ levels,

$$
\Phi \rightarrow \phi, \quad-i[,] \rightarrow \frac{1}{2}\{,\}, \quad \Lambda_{n} \rightarrow \lambda_{n}, \quad S \rightarrow \mathfrak{r} S, \quad \mathfrak{r}=\frac{\operatorname{deg}\left(\Lambda_{n}\right)+1}{\operatorname{deg}\left(\lambda_{n}\right)+1}
$$


Comment: it may naively appear that there exists a different DC prescription, one that maps the $n$-th YM family to the $(n-1)$-th gravity one. Upon relabeling the gravity subscripts, this would yield identical zeroth order expressions on both sides. However this prescription has various drawbacks. Firstly, it would map YM gauge symmetries $\delta_{1} \mathcal{A}_{\mu}=\delta_{\Lambda} \mathcal{A}_{\mu}=\partial_{\mu} \Lambda+i\left[\Lambda, \mathcal{A}_{\mu}\right]$ into trivial diffeomorphisms $\delta_{0} h_{\mu \nu}=0$. In particular, the large YM gauge symmetries at null infinity would not have a gravitational counterpart. Secondly, the second family of YM symmetries would be mapped to the first family of gravitational ones. As we shall see, this would imply an additional mismatch in the number of would-be DC related asymptotic symmetries. Finally, our original DC prescription (5.43) is shown in appendix $\mathrm{C}$ to be consistent with the convolution DC [43-45] where they overlap, whereas the alternative DC would not be.

\section{$6 \quad$ Large gauge symmetries at null infinity}

\subsection{Asymptotic fields at null infinity}

In this section we switch to coordinates adapted to null infinity. Given the light cone coordinates $(U, V, Z, \bar{Z})$ introduced in (2.1), a natural choice for Bondi-type coordinates $(r, u, z, \bar{z})$ is given by:

$$
U=r z \bar{z}+u, \quad V=r, \quad Z=r z, \quad \bar{Z}=r \bar{z},
$$

in terms of which the Minkowski line element takes the form

$$
d s^{2}=-2 d u d r+2 r^{2} d z d \bar{z} .
$$

Future null infinity $\mathcal{I}$ is reached by taking $r \rightarrow \infty$ while keeping the rest of the variables fixed. It is thus parametrized by $(u, z, \bar{z})$. Note that we will be working in a flat conformal frame for which the (degenerate) metric at null infinity is $2 d z d \bar{z} .{ }^{12}$

Massless fields are captured at null infinity by certain $r \rightarrow \infty$ leading components. For a massless scalar field, this is given by

$$
\varphi(r, u, z, \bar{z}) \stackrel{r \rightarrow \infty}{=} \varphi_{\mathcal{I}}(u, z, \bar{z}) / r+\cdots
$$

where the dots indicate terms that decay faster than $1 / r . \varphi_{\mathcal{I}}(u, z, \bar{z})$ is to be regarded as a field defined intrinsically at null infinity, which contains all the radiative data of $\varphi$. We will also refer to it as the 'free data' at $\mathcal{I}$, since it may be regarded as the analogue of the Cauchy data for the 'final time' hypersurface $\mathcal{I}$, see e.g. [64]. Similarly, gauge and metric fields at null infinity are captured by

$$
\begin{aligned}
& \mathcal{A}_{z}(r, u, z, \bar{z}) \stackrel{r \rightarrow \infty}{=} A_{z}(u, z, \bar{z})+\cdots \\
& h_{z z}(r, u, z, \bar{z}) \stackrel{r \rightarrow \infty}{=} r C_{z z}(u, z, \bar{z})+\cdots
\end{aligned}
$$

\footnotetext{
${ }^{12}$ This can be contrasted with the often used Bondi frame for which the $2 \mathrm{~d}$ metric is that of the unit sphere. See e.g. appendix A of [107] for a comparison between the two.
} 
and respective $z \leftrightarrow \bar{z}$ expressions. If we now consider expressions (2.10) and (2.11) in Bondi coordinates (6.1) we find

$$
\begin{array}{rlrl}
\mathcal{A}_{z} & =r \partial_{u} \Phi, & \mathcal{A}_{\bar{z}}=0, \\
h_{z z} & =r^{2} \partial_{u}^{2} \phi, & & h_{\bar{z} \bar{z}}=0,
\end{array}
$$

from which one easily finds how the gauge and gravity free data is encoded in the scalar fields free data (see (6.3))

$$
\begin{array}{rlrl}
A_{z} & =\partial_{u} \Phi_{\mathcal{I}}, & A_{\bar{z}} & =0 \\
C_{z z} & =\partial_{u}^{2} \phi_{\mathcal{I}}, \quad C_{\bar{z} \bar{z}}=0 .
\end{array}
$$

We thus recover the expected result that self-dual fields are associated with data at null infinity that has vanishing antiholomorphic components.

In the reminder of the section we will study how the 'bulk' symmetries described in the previous sections manifest at null infinity, with the aim of obtaining DC rules for asymptotic symmetries. Before getting started, however, it will be useful to briefly review the standard description of asymptotic symmetries.

\subsection{Review of large gauge symmetries at null infinity}

In the general (not necessarily self-dual) case, one considers gauge and diffeomorphism symmetries that are non-trivial at null infinity. In the YM case, these are generated by gauge parameters that asymptote to a non-trivial function on the celestial sphere $[68,108]$,

$$
\Lambda(r, u, z, \bar{z}) \stackrel{r \rightarrow \infty}{=} \Lambda_{0}(z, \bar{z})+\cdots
$$

where the dots denote terms that go to zero as $r \rightarrow \infty$. The induced symmetry transformation on the null infinity radiative data is then

$$
\delta_{\Lambda_{0}} A_{z}=\partial_{z} \Lambda_{0}+i\left[\Lambda_{0}, A_{z}\right] .
$$

In the gravitational case one finds two classes of vector fields: the Bondi-Metzner-Sachs supertranslations [61, 62], parametrized by functions $f(z, \bar{z})$,

$$
\xi_{f}=f(z, \bar{z}) \partial_{u}+\cdots
$$

and the Barnich-Troessaert superrotations [66], parametrized by holomorphic 2d vector fields $Y^{z}(z) \partial_{z}+Y^{\bar{z}}(\bar{z}) \partial_{\bar{z}}$,

$$
\xi_{Y}=Y^{z}(z) \partial_{z}+\frac{u}{2} \partial_{z} Y(z) \partial_{u}-\frac{1}{2} \partial_{z} Y(z) r \partial_{r}+(z \leftrightarrow \bar{z})+\cdots,
$$

where in both cases the dots denote terms that vanish when $r \rightarrow \infty$. The infinitesimal diffeomorphisms generated by these vector fields induce the following action on the null infinity radiative data:

$$
\begin{aligned}
& \delta_{f} C_{z z}=-2 \partial_{z}^{2} f+f \partial_{u} C_{z z} \\
& \delta_{Y} C_{z z}=-u \partial_{z}^{3} Y^{z}+\left(Y^{z} \partial_{z}+2 \partial_{z} Y^{z}+\frac{u}{2} \partial \cdot Y \partial_{u}-\frac{1}{2} \partial \cdot Y\right) C_{z z}
\end{aligned}
$$


where $\partial \cdot Y=\partial_{z} Y^{z}+\partial_{\bar{z}} Y^{\bar{z}}$. The transformation properties for $C_{\bar{z} \bar{z}}$ can be obtained by doing the replacement $z \rightarrow \bar{z}$ in the expressions above.

Whereas the above represent the simplest large gauge symmetries, it is possible to find additional ones by carefully relaxing the standard fall-off conditions (6.4), (6.5). In particular, one can make sense of gauge symmetries with $O(r)$ gauge parameter [109-112] and non-holomorphic superrotations [113-115]. Interestingly, the $O(r)$ gauge symmetries will turn out to play a key role in identifying the single copy version of superrotations, see section 7 .

Coming back to the self-dual case, we can ask what is the subset of the previously discussed symmetries that preserve the conditions

$$
A_{\bar{z}}=0, \quad C_{\bar{z} \bar{z}}=0
$$

at null infinity. On the YM side, this condition is preserved provided $\Lambda_{0}$ is holomorphic,

$$
\Lambda_{0}=\Lambda_{0}(z)
$$

On the gravity side, the supertranslation function has to be of the form

$$
f=f(z)+\bar{z} g(z)
$$

Finally, the antiholomorphic component of the superrotation vector field has to be of the form $Y^{\bar{z}}=a_{0}+a_{1} \bar{z}+a_{2} \bar{z}^{2}$ for some constants $a_{0}, a_{1}$ and $a_{2}$. This corresponds to Lorentz generators and is hence part of the global symmetry group. If, as in section 3 , we only keep track of local symmetries, we are left with vector fields of the form

$$
Y^{z}=Y(z), \quad Y^{\bar{z}}=0 .
$$

Below we will recover these restricted symmetries as the null infinity limit of the residual gauge symmetries discussed in section 3 .

\subsection{Residual gauge symmetries at null infinity}

We now discuss the null infinity limit of the residual gauge symmetries described in section 3. In order to do so, we will chose the functional form of the symmetry parameters that yields a finite and non-trivial action at null infinity.

Recall that the symmetry parameters can be constructed from functions which depend only on the $y^{\alpha}=(V, Z)$ variables: $\Lambda(y)$ for the YM gauge symmetries and $b(y)$ and $c(y)$ for the first and second family of gravitational symmetries. In terms of Bondi coordinates (6.1), this translates into functions of the variables $(r, z)$. We will consider simple functional forms, $F(r, z)=r^{n} F(z)$, and choose $n$ so as to obtain well defined $r \rightarrow \infty$ limits.

Let us start with the YM residual gauge symmetry (3.12). In order to have a welldefined action at null infinity, we need the r.h.s. of (3.12) to be $O\left(r^{-1}\right)$. For the homogeneous term, this can only be achieved if $\Lambda=O\left(r^{0}\right)$. We are thus led to choose $\Lambda$ as

$$
\Lambda(r, z)=\Lambda_{0}(z)
$$


Let us now discuss the inhomogeneous term in (3.12). To do so we note that the differential operator $S$ (5.33) in Bondi coordinates takes the form

$$
S \equiv \Omega_{i}{ }^{\alpha} x^{i} \partial_{\alpha}=-\bar{z} u \partial_{u}-\bar{z}^{2} \partial_{\bar{z}}+\bar{z} r \partial_{r}+\frac{u}{r} \partial_{z} .
$$

When (6.21) acts on $\Lambda$ (6.20), only the last term survives, yielding a $O\left(r^{-1}\right)$ result as expected. From here one can read off the action on the radiative data to be

$$
\delta_{\Lambda} \Phi_{\mathcal{I}}=u \partial_{z} \Lambda_{0}(z)+i\left[\Lambda_{0}(z), \Phi_{\mathcal{I}}\right]
$$

The corresponding action on $A_{z}$ can be obtained from the relation (6.8), where one recovers (6.11) for holomorphic $\Lambda_{0}$.

Consider now the first family of gravitational symmetries, (3.29), with $b_{\alpha}=\partial_{\alpha} b$ as in eq. (3.31). Writing the homogenous term in Bondi coordinates, one easily finds that, in order for this term to be $O\left(r^{-1}\right)$ one needs to take $b=O(r)$. We then choose

$$
b(r, z)=-r f(z) .
$$

The inhomogeneous term associated to $b$ turns out to be $O\left(r^{-1}\right)$ due to subtle cancellations. One finally arrives at

$$
\delta_{b} \phi_{\mathcal{I}}=-u^{2} f^{\prime \prime}(z)+f(z) \partial_{u} \phi_{\mathcal{I}}
$$

for the induced action on the free data. The corresponding action on $C_{z z}$ can be obtained from the relation (6.9), where one recovers (6.14) for $f=f(z)$. By similar considerations, one can extend the previous analysis to parameters $b_{\alpha}$ that are not necessarily a total derivative. One finds that, at null infinity, the only finite and non-trivial transformation that can be obtained is still of the form (6.24). Thus, the condition $b_{\alpha}=\partial_{\alpha} b$ does not imply a restriction as far as the asymptotic symmetries are concerned.

Let us finally discuss the second family of gravitational symmetries introduced in subsubsection 3.2.2. In this case one finds there are two possible choices of $c(y)$, either $O(r)$ or $O\left(r^{2}\right)$, that yield a finite and non-trivial null infinity limit:

$$
c(r, z)=r g(z) \quad \text { or } \quad c(r, z)=-r^{2} Y(z) / 2
$$

For the first choice, one obtains

$$
\delta_{c} \phi_{\mathcal{I}}=-u^{2} \bar{z} g^{\prime \prime}(z)+\bar{z} g(z) \partial_{u} \phi_{\mathcal{I}}
$$

which, upon translating to $C_{z z}$, yields a supertranslation (6.14) with $f(z, \bar{z})=\bar{z} g(z)$. For the second choice of $c$ in (6.25) one finds

$$
\delta_{c} \phi_{\mathcal{I}}=-\frac{u^{3}}{6} Y^{\prime \prime \prime}(z)+\left(Y(z) \partial_{z}+\frac{u}{2} Y^{\prime}(z) \partial_{u}+\frac{1}{2} Y^{\prime}(z)\right) \phi_{\mathcal{I}}
$$

which, when translated to $C_{z z}$ via (6.9), reproduces the superrotation action (6.15) with vector field (6.19). 


\section{Color to kinematic symmetry map at null infinity}

Having understood how large gauge symmetries emerge from the residual symmetries discussed in section 3, we now study how the DC for 'bulk' symmetries described in section 5 translates into a DC for asymptotic symmetries at null infinity. This will be achieved by studying the DC symmetry relations to leading order in a $1 / r$ expansion. Whereas for the first family (eqs. (5.34) and (5.35)) this is rather straightforward, for the second family (eqs. (5.37) and (5.38)) the expansion is trickier due to the appearance of the inverse wave operator. We will circumvent this difficulty by making use of the symmetry raising map discussed in section 5.4 .

To get started, let us express the operators that appear in the description of bulk symmetries in Bondi coordinates, paying special attention to their $1 / r$ expansion. Given a spacetime function of the form $r^{n} F_{n}(u, z, \bar{z})$, we will regard $F_{n}(u, z, \bar{z})$ as a function of "weight" $n$ at null infinity ( $n$ determines the transformation properties of $F_{n}(u, z, \bar{z})$ under a conformal rescaling). ${ }^{13}$

The PB in Bondi coordinates takes the form

$$
\{f, g\}=r^{-1}\{f, g\}_{\mathcal{I}}
$$

with

$$
\{f, g\}_{\mathcal{I}}=\left(\partial_{\bar{z}} f \partial_{u} g-\partial_{u} f \partial_{\bar{z}} g\right) .
$$

We will regard $\{,\}_{\mathcal{I}}$ as a PB defined intrinsically at null infinity (with weight -1 ). Consider now the differential operator

$$
S=\Omega_{i}{ }^{\alpha} x^{i} \partial_{\alpha}
$$

that features in the inhomogeneous part of the symmetries. Its form in Bondi coordinates was given in (6.21). When acting on a function of the form $r^{n} F_{n}(u, z, \bar{z})$, it can be written as:

$$
S\left(r^{n} F_{n}\right)=r^{n} S_{0}\left(F_{n}\right)+r^{n-1} S_{-1}\left(F_{n}\right)
$$

where

$$
\begin{aligned}
S_{0} & =-\bar{z} u \partial_{u}+n \bar{z}-\bar{z}^{2} \partial_{\bar{z}} \\
S_{-1} & =u \partial_{z} .
\end{aligned}
$$

$S_{0}$ and $S_{-1}$ will be regarded as differential operators defined intrinsically at null infinity. Note that $S_{0}$ depends on the weight $n$ of the function being acted upon.

We now proceed to describe the DC for the first and second family of asymptotic symmetries.

\subsection{First family}

The YM symmetry (5.34) at null infinity was described in eq. (6.22) for $\Lambda=\Lambda_{0}(z)$. Using the notation introduced above, we write it as

$$
\delta_{\Lambda} \Phi_{\mathcal{I}}=S_{-1}\left(\Lambda_{0}\right)+i\left[\Lambda_{0}, \Phi_{\mathcal{I}}\right]
$$

\footnotetext{
${ }^{13}$ Notice that this $n$ has nothing to do with the $n$ of section 5.5, which labeled the symmetry families.
} 
For the gravity symmetry (5.35), we need to compute the 'Hamiltonian' $\lambda$ (5.6) for the choice of $b(y)$ given in (6.23). This gives

$$
\lambda=2 S(b=-r f(z))=r \lambda_{1}+\lambda_{0}
$$

with

$$
\begin{aligned}
& \lambda_{1}=-2 S_{0}(f)=-2 \bar{z} f(z) \\
& \lambda_{0}=-2 S_{-1}(f)=-2 u f^{\prime}(z) .
\end{aligned}
$$

The homogenous term in (5.35) then has the expected order in $r$, since a $\lambda$ with an $O(r)$ piece will compensate for the $r^{-1}$ factor in the PB (7.1). Naively, it appears that the inhomogenous term could have $O(r)$ and $O\left(r^{0}\right)$ terms, thus spoiling the required $O\left(r^{-1}\right)$ fall-offs. However, such coefficients turn out to be zero. In the end one finds a well defined action on the radiative data, given by

$$
\delta \phi_{\mathcal{I}}=\frac{1}{2} S_{-1}\left(\lambda_{0}\right)-\frac{1}{2}\left\{\lambda_{1}, \phi_{\mathcal{I}}\right\}_{\mathcal{I}} .
$$

We emphasize that (7.11) is just a rewriting of (6.24), but one that makes the DC structure manifest. Comparing (7.7) to (7.11), we find that the DC rules at null infinity have the same structure as those in the bulk, as expected. The new ingredient is that, in the homogeneous term, the weight of the symmetry parameter increases by 1 (this is to compensate for the factor of $1 / r$ in the $\mathrm{PB}(7.1))$.

\subsection{Second family}

For the second family of symmetries, the DC relation in the bulk was made manifest by expressing the symmetries perturbatively, eqs. (5.37) and (5.38). Unfortunately, such expressions are difficult to expand in $1 / r$ due to the appearance of the inverse wave operator. However, the discussion given in section 5.4 offers an alternative route to obtaining the DC symmetry map: the second family of symmetries for each theory can be obtained from the first family via the "symmetry raising map", see the vertical arrows of (5.43). Thus, rather than going directly from $\delta_{\tilde{\Lambda}} \Phi$ to $\delta_{\tilde{\lambda}} \phi$ via the perturbative bulk DC map (bottom horizontal arrow of (5.43)), one can try to get there via the first family of symmetries. This is the strategy we will follow in this section to describe the second family of asymptotic symmetries and their DC relation.

Let us start with a general discussion that applies to both theories; for concreteness we only display the equations corresponding to the YM case. Since the first family of symmetries can be written as a sum of $\mathcal{O}\left(\Phi^{0}\right)$ and $\mathcal{O}\left(\Phi^{1}\right)$ terms, $\delta \Phi=\delta^{(0)} \Phi+\delta^{(1)} \Phi$, the raising map (2.18) produces a symmetry $\tilde{\delta}$ that is at most $\mathcal{O}\left(\Phi^{2}\right): \tilde{\delta} \Phi=\tilde{\delta}^{(0)} \Phi+\tilde{\delta}^{(1)} \Phi+\tilde{\delta}^{(2)} \Phi$. Collecting powers of $\Phi$ in (2.18), one finds each term should satisfy

$$
\begin{aligned}
\partial_{i} \tilde{\delta}^{(0)} \Phi & =\Omega_{i}{ }^{\alpha} \partial_{\alpha} \delta^{(0)} \Phi, \\
\partial_{i} \tilde{\delta}^{(1)} \Phi & =\Omega_{i}{ }^{\alpha} \partial_{\alpha} \delta^{(1)} \Phi-i\left[\partial_{i} \Phi, \delta^{(0)} \Phi\right], \\
\partial_{i} \tilde{\delta}^{(2)} \Phi & =-i\left[\partial_{i} \Phi, \delta^{(1)} \Phi\right] .
\end{aligned}
$$


Eq. (7.12) was already discussed in section 5.4, where it was found that

$$
\tilde{\delta}^{(0)} \Phi=\frac{1}{2} S^{2}(\Lambda)=\frac{1}{2} S(\tilde{\Lambda}), \quad \text { with } \quad \tilde{\Lambda}=\delta_{\Lambda}^{(0)} \Phi=S(\Lambda) .
$$

Similarly, if we start with a gravitational first family symmetry, so that $\delta^{(0)} \phi=\frac{1}{2} S(\lambda)$ (with $\lambda=2 S(b)$ ) one finds

$$
\tilde{\delta}^{(0)} \phi=\frac{1}{6} S^{2}(\lambda)=\frac{1}{3} S(\tilde{\lambda}), \quad \text { with } \quad \tilde{\lambda}=\delta_{\lambda}^{(0)} \phi=\frac{1}{2} S(\lambda) .
$$

The numerical factors in (7.15) and (7.16) arise as particular cases of the general coefficients explained in section 5.5. We will later expand these expressions in $1 / r$ in order to obtain $\tilde{\delta}^{(0)} \Phi_{\mathcal{I}}$ and $\tilde{\delta}^{(0)} \phi_{\mathcal{I}}$.

To obtain $\tilde{\delta}^{(1)} \Phi_{\mathcal{I}}$ and $\tilde{\delta}^{(2)} \Phi_{\mathcal{I}}$, we will solve eqs. (7.13) and (7.14) asymptotically as follows. First, we note that the $x^{i}$ derivatives expressed in Bondi coordinates,

$$
\begin{aligned}
& \partial_{U}=\partial_{u} \\
& \partial_{\bar{Z}}=-z \partial_{u}+r^{-1} \partial_{\bar{z}}
\end{aligned}
$$

are not independent in the $r \rightarrow \infty$ limit. Thus, to leading order in $1 / r$ it is enough to consider only one component of eqs. (7.13) and (7.14). Choosing $i=U$ and writing them in Bondi coordinates one obtains

$$
\begin{aligned}
\partial_{u} \tilde{\delta}^{(1)} \Phi & =\left(-\bar{z} \partial_{u}+r^{-1} \partial_{z}\right) \delta^{(1)} \Phi-i\left[\partial_{u} \Phi, \delta^{(0)} \Phi\right] \\
\partial_{u} \tilde{\delta}^{(2)} \Phi & =-i\left[\partial_{u} \Phi, \delta^{(1)} \Phi\right]
\end{aligned}
$$

In order to have a well defined action at null infinity, we need the r.h.s. of these equations to be $O\left(r^{-1}\right)$. The resulting $O\left(r^{-1}\right)$ factors will then give expressions for $\partial_{u} \tilde{\delta}^{(1)} \Phi_{\mathcal{I}}$ and $\partial_{u} \tilde{\delta}^{(2)} \Phi_{\mathcal{I}}$, from which the final answer can be obtained by a single integral in $u$.

Similar considerations apply to the gravitational case, in which case $\tilde{\delta}^{(1)} \phi_{\mathcal{I}}, \tilde{\delta}^{(2)} \phi_{\mathcal{I}}$ are to be determined from the $O\left(r^{-1}\right)$ part of the equations

$$
\begin{aligned}
\partial_{u} \tilde{\delta}^{(1)} \phi & =\left(-\bar{z} \partial_{u}+r^{-1} \partial_{z}\right) \delta^{(1)} \phi+\frac{1}{2}\left\{\partial_{u} \phi, \delta^{(0)} \phi\right\} \\
\partial_{u} \tilde{\delta}^{(2)} \phi & =\frac{1}{2}\left\{\partial_{u} \phi, \delta^{(1)} \phi\right\} .
\end{aligned}
$$

In order to perform the required $1 / r$ expansion, we need to specify the asymptotic behavior of the symmetry parameters $\Lambda$ and $\lambda$. We now describe two possibilities that yield a well defined action of $\tilde{\delta}$ at null infinity.

\subsection{1 $\tilde{\delta}$ from $\Lambda=O\left(r^{0}\right), \lambda=O(r)$}

We start by considering symmetry parameters as those discussed in section 7.1,

$$
\begin{aligned}
\Lambda & =\Lambda_{0}(z) \\
\lambda & =r \lambda_{1}+\lambda_{0}
\end{aligned}
$$


with $\lambda$ as in eqs. (7.8), (7.9), (7.10). The corresponding $\tilde{\Lambda}$ and $\tilde{\lambda}$ defined in eqs. (7.15) and (7.16) take the form

$$
\tilde{\Lambda}=r^{-1} \tilde{\Lambda}_{-1}, \quad \tilde{\lambda}=r^{-1} \tilde{\lambda}_{-1}
$$

with

$$
\begin{aligned}
& \tilde{\Lambda}_{-1}=S_{-1}\left(\Lambda_{0}\right)=u \Lambda_{0}^{\prime}(z) \\
& \tilde{\lambda}_{-1}=\frac{1}{2} S_{-1}\left(\lambda_{0}\right)=-u^{2} f^{\prime \prime}(z) .
\end{aligned}
$$

One then obtains

$$
\begin{aligned}
\tilde{\delta}^{(0)} \Phi_{\mathcal{I}} & =\frac{1}{2} S_{0}\left(\tilde{\Lambda}_{-1}\right)=-u \bar{z} \Lambda_{0}^{\prime}(z) \\
\tilde{\delta}^{(0)} \phi_{\mathcal{I}} & =\frac{1}{3} S_{0}\left(\tilde{\lambda}_{-1}\right)=u^{2} \bar{z} f^{\prime \prime}(z)
\end{aligned}
$$

for the inhomogeneous part of the symmetry transformations. The linear part of the symmetries can be obtained from eqs. (7.19), (7.21), taking into account the form of $\delta^{(0)}$ and $\delta^{(1)}$ given in section 7.1. One finds that the only terms contributing to order $O\left(r^{-1}\right)$ are those coming from the $\bar{z} \partial_{u}$ term hitting the commutator/PB. This allows for a trivial integration in $u$, leading to

$$
\begin{aligned}
\tilde{\delta}^{(1)} \Phi_{\mathcal{I}} & =-i \bar{z}\left[\Lambda_{0}, \Phi_{\mathcal{I}}\right] \\
\tilde{\delta}^{(1)} \phi_{\mathcal{I}} & =\frac{1}{2} \bar{z}\left\{\lambda_{1}, \phi_{\mathcal{I}}\right\}_{\mathcal{I}} .
\end{aligned}
$$

Finally, one finds that the r.h.s. of eqs. (7.20) and (7.22) decay faster than $O\left(r^{-1}\right)$ and hence

$$
\tilde{\delta}^{(2)} \Phi_{\mathcal{I}}=0, \quad \tilde{\delta}^{(2)} \phi_{\mathcal{I}}=0 .
$$

The gravitational symmetry $\tilde{\delta} \phi_{\mathcal{I}}$ can be seen to coincide with a supertranslation associated to $\tilde{f}(z, \bar{z})=-\bar{z} f(z)$ (eq. (6.26) with $g(z) \rightarrow f(z)$ ). Thus, the symmetry raising map takes a supertranslation with $f=f(z)$ into a supertranslation with $\tilde{f}=-\bar{z} f(z)$.

\subsection{2 $\tilde{\delta}$ from $\Lambda=O(r), \lambda=O\left(r^{2}\right)$}

We now consider symmetry parameters $\Lambda$ and $\lambda$ of one order higher in $r$. Although these generate transformations that violate the $r \rightarrow \infty$ fall-offs of the YM and gravity fields, the corresponding $\tilde{\delta}$ symmetries will turn out to preserve such fall-offs.

On the YM side, we consider a $O(r)$ gauge transformation

$$
\Lambda=r \Lambda_{1}(z)+\Lambda_{0}
$$

where $\Lambda_{0}=u \partial_{z} \partial_{\bar{z}} \Lambda_{1}(z)=0$ (as obtained from the condition $\square \Lambda=0$ ). We explicitly keep this vanishing term in (7.33) since it will get mapped into a non-trivial term under the DC. The corresponding $\tilde{\Lambda}$ is:

$$
\tilde{\Lambda}=S(\Lambda)=r S(\Lambda)_{1}+S(\Lambda)_{0}
$$


with $^{14}$

$$
\tilde{\Lambda}_{1}=S(\Lambda)_{1}=S_{0}\left(\Lambda_{1}\right)=\bar{z} \Lambda_{1}(z), \quad \tilde{\Lambda}_{0}=S(\Lambda)_{0}=S_{-1}\left(\Lambda_{1}\right)=u \Lambda_{1}^{\prime}(z) .
$$

Substituting this expansion in $(7.15)$ one finds ${ }^{15}$

$$
\tilde{\delta}^{(0)} \Phi_{\mathcal{I}}=\frac{1}{2} S_{-1}\left(\tilde{\Lambda}_{0}\right)=\frac{u^{2}}{2} \Lambda_{1}^{\prime \prime}(z) .
$$

To obtain $\tilde{\delta}^{(1)} \Phi_{\mathcal{I}}$, we consider eq. (7.19) for $\delta_{\Lambda}^{(0)} \Phi=S(\Lambda)$ and $\delta_{\Lambda}^{(1)} \Phi=i[\Lambda, \Phi]$. Interestingly, the potentially divergent terms cancel out, and one arrives at a finite equation for the null infinity free data $\Phi_{\mathcal{I}}$ :

$$
\partial_{u} \tilde{\delta}^{(1)} \Phi_{\mathcal{I}}=-i \bar{z} \partial_{u}\left[\Lambda_{0}, \Phi_{\mathcal{I}}\right]+i \partial_{z}\left[\Lambda_{1}, \Phi_{\mathcal{I}}\right]-i\left[\partial_{u} \Phi_{\mathcal{I}}, S_{-1}\left(\Lambda_{1}\right)\right]
$$

Similarly, from eq. (7.20) one obtains

$$
\partial_{u} \tilde{\delta}^{(2)} \Phi_{\mathcal{I}}=\left[\partial_{u} \Phi_{\mathcal{I}},\left[\Lambda_{1}, \Phi_{\mathcal{I}}\right]\right]
$$

On the gravitational side, we now consider a symmetry parameter $b$ that is $O\left(r^{2}\right)$

$$
b=-r^{2} Y(z) / 2 .
$$

The corresponding $\lambda=2 S(b)$ takes the form

$$
\lambda=r^{2} \lambda_{2}+r \lambda_{1}
$$

with

$$
\lambda_{2}=-2 \bar{z} Y(z), \quad \lambda_{1}=-u Y^{\prime}(z) .
$$

$S(\lambda)=2 \tilde{\lambda}$ is then given by

$$
S(\lambda)=r^{2} S(\lambda)_{2}+r S(\lambda)_{1}+S(\lambda)_{0}
$$

with

$$
\begin{aligned}
& S(\lambda)_{2}=S_{0}\left(\lambda_{2}\right)=-2 \bar{z}^{2} Y(z), \\
& S(\lambda)_{1}=S_{-1}\left(\lambda_{2}\right)=-2 u \bar{z} Y^{\prime}(z), \\
& S(\lambda)_{0}=S_{-1}\left(\lambda_{1}\right)=-u^{2} Y^{\prime \prime}(z),
\end{aligned}
$$

where we used that $S_{0}\left(\lambda_{1}\right)=0$. To obtain $\tilde{\delta}^{(0)} \phi_{\mathcal{I}}$, we substitute the above expansion in (7.16). It turns out that all potentially divergent terms cancel out and one is left with a $O\left(r^{-1}\right)$ term, yielding

$$
\tilde{\delta}^{(0)} \phi_{\mathcal{I}}=\frac{1}{3} S_{-1}\left(\tilde{\lambda}_{0}\right)=-\frac{u^{3}}{6} Y^{\prime \prime \prime}(z) .
$$

\footnotetext{
${ }^{14}$ Since $S(\Lambda)$ has a non-trivial $O\left(r^{0}\right)$ part, there is no need in this case to explicitly include the vanishing $\Lambda_{0}$ contribution.

${ }^{15}$ The structure of $\tilde{\Lambda}$ in this case is formally identical to the $\lambda$ of the first gravitational symmetry (7.8), with $\Lambda_{1}=-2 f$. The same cancellations occur when computing $S(\tilde{\Lambda})$, so that only the $O\left(r^{-1}\right)$ part survives.
} 
This precisely reproduces the inhomogenous part of the superrotation action (6.27).

To obtain $\tilde{\delta}^{(1)} \phi_{\mathcal{I}}$, we consider eq. (7.21) for $\delta_{\lambda}^{(0)} \phi=\frac{1}{2} S(\lambda)$ and $\delta_{\lambda}^{(1)} \phi=-\frac{1}{2}\{\lambda, \phi\}$, with $\lambda, S(\lambda)$ as given by (7.40), (7.42). Once again the divergent terms cancel out, and one obtains a well defined equation at null infinity:

$$
\partial_{u} \tilde{\delta}^{(1)} \phi_{\mathcal{I}}=\frac{1}{2} \bar{z} \partial_{u}\left\{\lambda_{1}, \phi_{\mathcal{I}}\right\}_{\mathcal{I}}-\frac{1}{2} \partial_{z}\left\{\lambda_{2}, \phi_{\mathcal{I}}\right\}_{\mathcal{I}}+\frac{1}{4}\left\{\partial_{u} \phi_{\mathcal{I}}, S_{-1}\left(\lambda_{2}\right)\right\}_{\mathcal{I}}
$$

This is the DC version of (7.37), with the rule described in section 7.1 for the increase in weight when going from $\Lambda$ to $\lambda$, and the multiplicative $\mathfrak{r}$ factor associated to the operator $S$, see equation (5.36). Finally, the r.h.s. of (7.20) is found to be $O\left(r^{-2}\right)$ and hence

$$
\tilde{\delta}^{(2)} \phi_{\mathcal{I}}=0 \text {. }
$$

It may be puzzling that the non-zero quadratic term (7.38) trivializes after the DC. From the perspective of null infinity, this happens because the DC takes the weight 0 commutator [,] into the weight -1 Poisson bracket $\{,\}_{\mathcal{I}}$. Since in both cases one should have overall weight minus one, one would need to map $\Lambda_{1}$ into a weight $3 \lambda_{3}$ to get a non-trivial DC result. However there is no $\lambda_{3}$ in the gravitational symmetry under consideration.

We conclude by noting that if one expands (7.47) by explicitly writing the PB (7.2) and the parameters $(7.41),(7.44)$, one recovers, as expected, the total $u$-derivative of the homogenous part of the superrotation action (6.27). Thus, the symmetry raising map takes a "divergent supertranslation" defined by (7.39) into a superrotation.

\subsection{Summary}

In figure 1 we summarize the different symmetries at null infinity, together with the functional form of their parameters. The diagram may be thought of as an augmented version of (5.43), with a new layer due to the two possibilities that arise in the second family of symmetries. In the first line we have holomorphic non-abelian large gauge transformations (6.22) and holomorphic supertranslations (6.24). In the second line we have the non-gauge YM transformation (7.28), (7.30) and the second type of allowed supertranslations (6.26). The third line displays the non-gauge YM transformation defined by eqs. (7.36), (7.37), (7.38) and the superrotations (6.27).

The horizontal arrows represent the double copy relations between the different symmetries. The replacement rules are as in the bulk, with the following clarification: the commutator is replaced by the PB at null infinity (7.2), which has weight -1 . To compensate for this, YM parameters of weight $n$ are replaced with Hamiltonians of weight $n+1$.

Vertical arrows describe the symmetry raising map, see section 5.4. Diagonal arrows describe the increase in the power of $r$ associated to the parameter of the second family of symmetries, see e.g. eq. (6.25) for the gravitational case. To better understand these arrows, one can imagine completing the diagram by raising the power of $r$ in the parameters of the first family of YM and gravity symmetries, thus completing the cube. We can then recover the bottom two symmetries by applying the symmetry raising map to these new vertices, see subsubsection 7.2.2 for details. We chose not to display them in the diagram since they do not preserve the $r \rightarrow \infty$ field fall-offs. ${ }^{16}$

\footnotetext{
${ }^{16}$ See the discussion section for further comments on this point.
} 


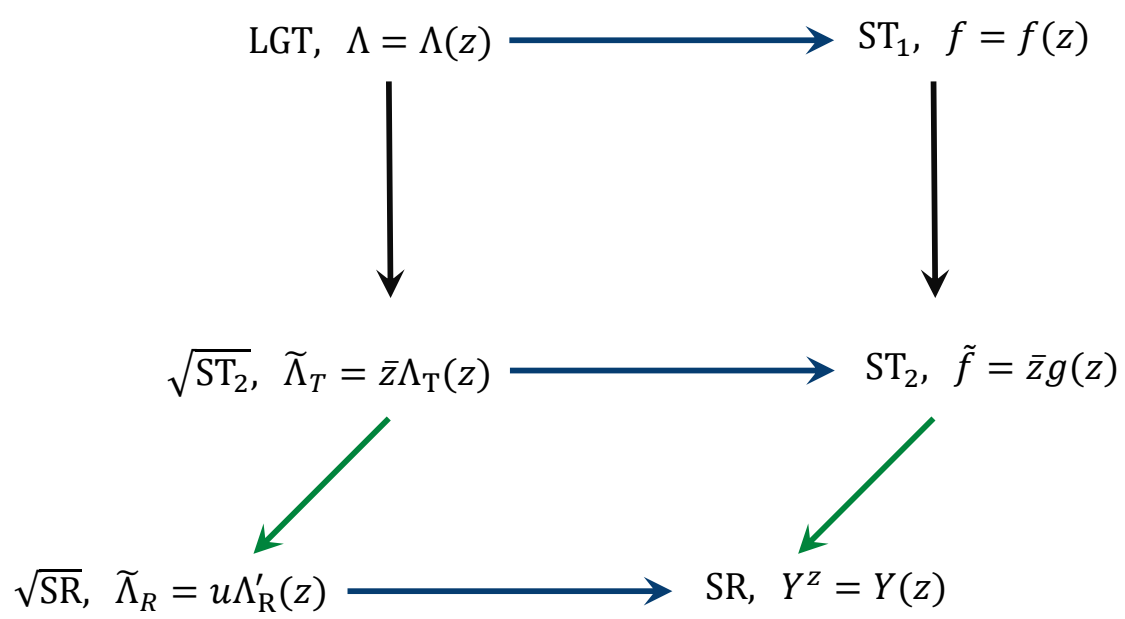

Figure 1. Summary of the asymptotic symmetries and their various relations. LGT stands for non-abelian large gauge transformations, ST and SR for supertranslations and superrotations. Horizontal lines represent the DC relations. Vertical lines represent the symmetry raising map between the first and second family of symmetries. Diagonal arrows represent an increase in the power of $r$ of symmetry parameters, see main text for details.

\section{Discussion}

In this paper we presented the double copy construction of a subset of asymptotic symmetries. Our starting point was the study of residual symmetries of the self-dual sectors of YM and gravity, in the light-cone formulation. We identified two families of symmetries. For the first one, we found novel non-pertubative double copy rules in the bulk, leading, in the asymptotic regime, to the mapping of a holomorphic YM symmetry to a holomorphic supertranslation. The second family has a more striking structure, in the sense that an exact subset of diffeomorphism symmetries is obtained as the double copy of a perturbatively-defined and non-local transformation in the YM self-dual sector. At null infinity, we identify the YM origin of a subset of superrotations with a novel symmetry transformation, which to our knowledge has not been previously presented in the literature.

An important open problem is how to extend our DC symmetry prescription beyond the self-dual context. Promising avenues for extending the Monteiro and O'Connell formulation have been given in e.g. [88, 89]. Another possibility would be to make use of the techniques developed in [116], where the full BMS algebra is obtained from the residual symmetries of the gravitational theory in the light-cone gauge. It would also be interesting to approach the problem via the formalism developed in [117]. Alternatively, one could try to extend our analysis to other families of solutions for which the doubly copy is manifest. An obvious first step would be to look at solutions obtained from the Kerr-Schild ansatz [19], which is structurally similar to the self-dual metric ansatz used in this paper.

The self-dual theories are known to possess infinitely many symmetries. An infinite tower of such symmetries can be obtained by iterating the 'symmetry raising map' reviewed in eqs. (2.18), (2.19). From this perspective, the two families of symmetries described above represent just the first two rungs of an infinite ladder. Whereas we have demonstrated that 
DC relations in the bulk are obeyed at each level, we have not yet explored the realization of these higher level symmetries at null infinity. It is tempting to speculate that these would be related, in the scattering amplitudes context, to the remarkable simplicity of Maximally Helicity Violating (MHV) amplitudes. This expectation is justified by existing explanations of MHV formulae based on the integrability properties of the self-dual equations, see e.g. $[101,118,119]$.

It is worthwhile to point out that, asymptotically, there is potentially yet another hierarchy if one allows for gauge transformations that diverge as some power of $r$ [109, 120-124]. There appears to be an interesting interplay between this hierarchy and the one described in the previous paragraph. Indeed, we were lead to consider $O(r)$ divergent gauge symmetries in order to fully implement the raising map between the first two families of asymptotic symmetries. It may be that the higher level families are related to gauge transformations diverging with higher powers of $r$.

\section{Acknowledgments}

We would like to thank Alok Laddha for illuminating discussions. MC acknowledges support from PEDECIBA and from ANII grant FCE-1-2019-1-155865. SN is supported by STFC grant ST/T000686/1. This research was supported by the Munich Institute for Astro- and Particle Physics (MIAPP) which is funded by the Deutsche Forschungsgemeinschaft (DFG, German Research Foundation) under Germany‘s Excellence Strategy EXC-2094 - 390783311.

\section{A Self-duality conditions}

\section{A.1 Yang-Mills field}

Given the field strength

$$
F_{\mu \nu}=\partial_{\mu} \mathcal{A}_{\nu}-\partial_{\nu} \mathcal{A}_{\mu}-i\left[\mathcal{A}_{\mu}, \mathcal{A}_{\mu}\right]
$$

its dual is defined as

$$
\tilde{F}_{\mu \nu}:=\frac{1}{2} \epsilon_{\mu \nu}{ }^{\rho \sigma} F_{\rho \sigma} .
$$

We say the field strength is self-dual if

$$
\tilde{F}_{\mu \nu}=i F_{\mu \nu}
$$

In the notation of $\left(x^{i}, y^{\alpha}\right)$ coordinates, the independent components of the volume form $\epsilon_{\mu \nu \rho \sigma}$ are

$$
\epsilon_{\alpha \beta i j}=i \Pi_{\alpha \beta} \Omega_{i j}
$$

The field strength of the YM field (2.8) is given by

$$
\begin{aligned}
F_{i j} & =0 \\
F_{i \alpha} & =\Pi_{\alpha}^{j} \partial_{i} \partial_{j} \Phi \\
F_{\alpha \beta} & =2 \Pi_{[\alpha}^{i} \partial_{\beta]} \partial_{i} \Phi-i \Pi_{\alpha}^{i} \Pi_{\beta}^{j}\left[\partial_{i} \Phi, \partial_{j} \Phi\right]
\end{aligned}
$$


It is easy to see that $\tilde{F}_{i j}=0$. Using (2.6) one can verify that $\tilde{F}_{i \alpha}=i F_{i \alpha}$. Let us then focus on $F_{\alpha \beta}$. Since it is antisymmetric in the $2 \mathrm{~d}$ indices $(\alpha, \beta)$, it can be written as multiple of $\Pi_{\alpha \beta}$ :

$$
F_{\alpha \beta}=f \Pi_{\alpha \beta} \quad \text { with } \quad f=-\frac{1}{2} \Omega^{\alpha \beta} F_{\alpha \beta}
$$

The spacetime dual is then found to be given by $\tilde{F}_{\alpha \beta}=-i F_{\alpha \beta}$. Thus, the only way to satisfy the selfdual condition (A.3) is that this component vanishes:

$$
F_{\alpha \beta}=0 \Longleftrightarrow \Omega^{\alpha \beta} F_{\alpha \beta}=0 .
$$

From (A.7) and using (2.6) one finds

$$
\Omega^{\alpha \beta} F_{\alpha \beta}=-\left(\square \Phi+i \Pi^{i j}\left[\partial_{i} \Phi, \partial_{j} \Phi\right]\right)
$$

so that indeed it vanishes provided (2.12) is satisfied.

\section{A.2 Metric field}

For spacetime metrics, the dual of the curvature tensor is defined as:

$$
\tilde{R}_{\mu \nu \rho}{ }^{\sigma}:=\frac{1}{2} \epsilon_{\mu \nu}{ }^{\eta \lambda} R_{\eta \lambda \rho}{ }^{\sigma},
$$

and we say the metric is self-dual if

$$
\tilde{R}_{\mu \nu \rho}{ }^{\sigma}=i R_{\mu \nu \rho}{ }^{\sigma} .
$$

We now study this condition for the spacetime metric

$$
g_{\mu \nu}=\eta_{\mu \nu}+h_{\mu \nu}
$$

with $h_{\mu \nu}$ given by (2.9). Note that we are not requiring $h$ to be a perturbation, and regard (A.13) as a full, non-linear metric. The form of $\eta$ and $h$ imply the inverse metric is given exactly by

$$
g^{\mu \nu}=\eta^{\mu \nu}-h^{\mu \nu} .
$$

One can also check that the determinant of $g$ coincides with that of $\eta$. In particular, the volume form is still given by (A.4). The non-zero Christoffel symbols are:

$$
\begin{aligned}
& \Gamma_{i \alpha}^{j}=\frac{1}{2} \eta^{\beta j} \partial_{i} h_{\alpha \beta}, \quad \Gamma_{\alpha \beta}^{i}=\frac{1}{2} \eta^{\gamma i}\left(\partial_{\alpha} h_{\beta \gamma}+\partial_{\beta} h_{\alpha \gamma}-\partial_{\gamma} h_{\alpha \beta}\right)+\frac{1}{2} h^{i j} \partial_{j} h_{\alpha \beta}, \\
& \Gamma_{\alpha \beta}^{\gamma}=-\frac{1}{2} \eta^{\gamma i} \partial_{i} h_{\alpha \beta}
\end{aligned}
$$

These expressions lead to the following independent components of the curvature tensor:

$$
\begin{aligned}
& R_{i j k}^{l}=0 \\
& R_{i j k}{ }^{\alpha}=0 \\
& R_{i \alpha j}{ }^{k}=-\frac{1}{2} \eta^{\beta k} \partial_{i} \partial_{j} h_{\alpha \beta} \\
& R_{\alpha \beta \gamma}{ }^{\delta}=\frac{1}{2} \eta^{\delta i} \partial_{i} \partial_{\alpha} h_{\beta \gamma}+\frac{1}{4} \eta^{\delta i} \eta^{\epsilon j} \partial_{j} h_{\alpha \gamma} \partial_{i} h_{\beta \epsilon}-(\alpha \leftrightarrow \beta) \\
& R_{\alpha \beta \gamma}{ }^{i}=\partial_{\beta} \Gamma_{\alpha \gamma}^{i}+\Gamma_{\alpha \gamma}^{\delta} \Gamma_{\delta \beta}^{i}+\Gamma_{\alpha \gamma}^{j} \Gamma_{j \beta}^{i}-(\alpha \leftrightarrow \beta)
\end{aligned}
$$


We now consider the dual curvature tensor (A.12). Here it is important to note that the raising of indices of the volume form must be realized with the full inverse metric (A.14). We will however continue to raise and lower indices on $\Pi$ and $\Omega$ with the flat metric $\eta$.

It is easy to check that $\tilde{R}_{i j k}{ }^{l}=\tilde{R}_{i j k}{ }^{\alpha}=0$, as well as $\tilde{R}_{i \alpha j}{ }^{k}=i R_{i \alpha j}{ }^{k}$. We now discuss the self-dual condition for the components (A.19). Since the expression is antisymmetric in the $2 \mathrm{~d} \alpha, \beta$ indices, we can contract with $\Omega^{\alpha \beta}$. After some algebra, one finds

$$
\Omega^{\alpha \beta}\left(\tilde{R}_{\alpha \beta \gamma}^{\delta}-i R_{\alpha \beta \gamma}{ }^{\delta}\right)=-i \Pi_{\gamma}^{m} \eta^{\delta n} \partial_{m} \partial_{n} \mathrm{E}_{\phi}
$$

with

$$
\mathrm{E}_{\phi}=\square \phi-\frac{1}{2} \Pi^{i j} \Pi^{k l} \partial_{i} \partial_{k} \phi \partial_{j} \partial_{l} \phi
$$

where $\mathrm{E}_{\phi}=0$ is the SDE equation (2.13). Finally, the self-dual condition for (A.20), contracted with $\Omega^{\alpha \beta}$, gives

$$
\Omega^{\alpha \beta}\left(\tilde{R}_{\alpha \beta \gamma}{ }^{i}-i R_{\alpha \beta \gamma}{ }^{i}\right)=i \Pi^{i j} \partial_{j} \partial_{\gamma} \mathrm{E}_{\phi}-i \eta^{i \alpha} \Pi_{\gamma}^{j} \partial_{j} \partial_{\alpha} \mathrm{E}_{\phi}+i \Pi^{j k} \Pi_{\gamma}^{l} \Pi^{i m} \partial_{j} \partial_{l} \mathrm{E}_{\phi} \partial_{k} \partial_{m} \phi .
$$

\section{B Perturbative transformations at higher orders}

In a perturbative setting, the double copy rules (5.36) can be written as:

$$
\Phi^{(i)} \rightarrow \phi^{(i)}, \quad-i[,] \rightarrow \frac{1}{2}\{,\}, \quad \Lambda \rightarrow \lambda, \quad \mathfrak{r}=\frac{\operatorname{deg}(\Lambda)+1}{\operatorname{deg}(\lambda)+1}
$$

where

$$
\begin{aligned}
& \Phi=\Phi^{(0)}+\Phi^{(1)}+\cdots \\
& \phi=\phi^{(0)}+\phi^{(1)}+\cdots
\end{aligned}
$$

\section{B.1 Second order}

\section{B.1.1 First family}

The first family of symmetries was shown to double copy non-perturbatively in section 5.1. However, as a warm-up, we demonstrate the perturbative construction to second order in perturbation theory, before proceeding to the second family. Working to linear order, the first family of transformations acts on the YM scalar as (see (4.9) and (4.13)):

$$
\begin{aligned}
& \delta_{\Lambda} \Phi^{(0)}=\Omega_{j}{ }^{\beta} x^{j} \partial_{\beta} \Lambda, \\
& \delta_{\Lambda} \Phi^{(1)}=-i\left[\Phi^{(0)}, \Lambda\right]
\end{aligned}
$$

We will now treat these as seeds and use the e.o.m. at second order in $\Phi$ :

$$
\square \Phi^{(2)}=-2 i \Pi^{i j}\left[\partial_{i} \Phi^{(0)}, \partial_{j} \Phi^{(1)}\right]
$$

to derive $\delta_{\Lambda} \Phi^{(2)}$. We have

$$
\begin{aligned}
\square \delta_{\Lambda} \Phi^{(2)} & =-2 i \Pi^{i j}\left[\partial_{i} \delta_{\Lambda} \Phi^{(0)}, \partial_{j} \Phi^{(1)}\right]-2 i \Pi^{i j}\left[\partial_{i} \Phi^{(0)}, \partial_{j} \delta_{\Lambda} \Phi^{(1)}\right] \\
& =-2 i \Pi^{i j}\left[\Omega_{i}{ }^{\beta} \partial_{\beta} \Lambda, \partial_{j} \Phi^{(1)}\right]-2 \Pi^{i j}\left[\partial_{i} \Phi^{(0)},\left[\partial_{j} \Phi^{(0)}, \Lambda\right]\right] \\
& =i \square\left[\Lambda, \Phi^{(1)}\right]-i\left[\Lambda, \square \Phi^{(1)}\right]+\Pi^{i j}\left[\Lambda,\left[\partial_{i} \Phi^{(0)}, \partial_{j} \Phi^{(0)}\right]\right]
\end{aligned}
$$


where we used the Jacobi identity for the commutator and the fact that $\Lambda=\Lambda(y)$. Then, using the eom for $\Phi^{(1)}$, we are left with

$$
\delta_{\Lambda} \Phi^{(2)}=-i\left[\Phi^{(1)}, \Lambda\right]
$$

Next, we look at the gravity transformation. Our seeds can be obtained by perturbing (5.7):

$$
\begin{aligned}
\delta_{\lambda} \phi^{(0)} & =\frac{1}{2} \Omega_{i}{ }^{\alpha} x^{i} \partial_{\alpha} \lambda \\
\delta_{\lambda} \phi^{(1)} & =\frac{1}{2}\left\{\phi^{(0)}, \lambda\right\}
\end{aligned}
$$

We will now use the e.o.m. at second order in $\phi$,

$$
\square \phi^{(2)}=\Pi^{i j} \Pi^{k l} \partial_{i} \partial_{k} \phi^{(0)} \partial_{j} \partial_{l} \phi^{(1)}
$$

to derive $\delta_{\lambda} \phi^{(2)}$. We have

$$
\begin{aligned}
\square \delta_{\lambda} \phi^{(2)} & =\frac{1}{2} \Pi^{i j} \Pi^{k l} \partial_{i} \partial_{k} \delta_{\lambda} \phi^{(0)} \partial_{j} \partial_{l} \phi^{(1)}+\frac{1}{2} \Pi^{i j} \Pi^{k l} \partial_{i} \partial_{k} \phi^{(0)} \partial_{j} \partial_{l} \delta_{\lambda} \phi^{(1)} \\
& =\Pi^{i j} \Pi^{k l} \partial_{i}\left(\Omega_{k}^{\alpha} \partial_{\alpha} \lambda\right) \partial_{j} \partial_{l} \phi^{(1)}+\frac{1}{2} \Pi^{i j} \Pi^{k l} \partial_{i} \partial_{k} \phi^{(0)} \partial_{j}\left\{\partial_{l} \phi^{(0)}, \lambda\right\} \\
& =-\eta^{l \alpha} \Pi^{i j} \partial_{i} \partial_{\alpha} \lambda \partial_{j} \partial_{l} \phi^{(1)}-\frac{1}{4} \Pi^{k l}\left\{\lambda,\left\{\partial_{k} \phi^{(0)}, \partial_{l} \phi^{(0)}\right\}\right\} \\
& =-\frac{1}{2} \square\left\{\tilde{\lambda}, \phi^{(1)}\right\}+\frac{1}{2}\left\{\tilde{\lambda}, \square \phi^{(1)}\right\}-\frac{1}{4} \Pi^{k l}\left\{\tilde{\lambda},\left\{\partial_{k} \phi^{(0)}, \partial_{l} \phi^{(0)}\right\}\right\}
\end{aligned}
$$

where we used the Jacobi identity for the Poisson bracket and the fact that $\lambda$ is linear in $x$. Then, using the e.o.m. for $\phi^{(1)}$ we get

$$
\delta_{\lambda} \phi^{(2)}=\frac{1}{2}\left\{\phi^{(1)}, \lambda\right\}
$$

and, comparing with (B.6), we see that it is obtained from $\delta_{\Lambda} \Phi^{(2)}$ via the double copy rules (B.1).

\section{B.1.2 Second family}

Here we extend the construction of the second family of symmetries and their double copy to second order in perturbation theory. Working to linear order, the second family of transformations acts on the YM scalar as (see (5.22) and (5.25))

$$
\begin{aligned}
& \delta_{\tilde{\Lambda}} \Phi^{(0)}=\frac{1}{2} \Omega_{i}{ }^{\alpha} x^{i} \partial_{\alpha} \tilde{\Lambda}, \\
& \delta_{\tilde{\Lambda}} \Phi^{(1)}=-i\left[\Phi^{(0)}, \tilde{\Lambda}\right]+2 i \square^{-1} \eta^{i \alpha}\left[\partial_{\alpha} \Phi^{(0)}, \partial_{i} \tilde{\Lambda}\right]
\end{aligned}
$$

We will now treat these as seeds and use the e.o.m. at second order in $\Phi$ :

$$
\square \Phi^{(2)}=-2 i \Pi^{i j}\left[\partial_{i} \Phi^{(0)}, \partial_{j} \Phi^{(1)}\right]
$$


to derive $\delta_{\tilde{\Lambda}} \Phi^{(2)}$. We have

$$
\begin{aligned}
\square \delta_{\tilde{\Lambda}} \Phi^{(2)}= & -2 i \Pi^{i j}\left[\partial_{i} \delta_{\tilde{\Lambda}} \Phi^{(0)}, \partial_{j} \Phi^{(1)}\right]-2 i \Pi^{i j}\left[\partial_{i} \Phi^{(0)}, \partial_{j} \delta_{\tilde{\Lambda}} \Phi^{(1)}\right] \\
= & -2 i \Pi^{i j}\left[\Omega_{i}{ }^{\alpha} \partial_{\alpha} \tilde{\Lambda}, \partial_{j} \Phi^{(1)}\right]-2 \Pi^{i j}\left[\partial_{i} \Phi^{(0)},\left[\partial_{j} \Phi^{(0)}, \tilde{\Lambda}\right]\right]-2 \Pi^{i j}\left[\partial_{i} \Phi^{(0)},\left[\Phi^{(0)}, \partial_{j} \tilde{\Lambda}\right]\right] \\
& +4 \Pi^{i j}\left[\partial_{i} \Phi^{(0)}, \partial_{j} \square^{-1} \eta^{k \alpha}\left[\partial_{\alpha} \Phi^{(0)}, \partial_{k} \tilde{\Lambda}\right]\right] \\
= & 2 i \eta^{i \alpha}\left[\partial_{\alpha} \tilde{\Lambda}, \partial_{i} \Phi^{(1)}\right]+\Pi^{i j}\left[\tilde{\Lambda},\left[\partial_{i} \Phi^{(0)}, \partial_{j} \Phi^{(0)}\right]\right]-2 \Pi^{i j}\left[\partial_{i} \Phi^{(0)},\left[\Phi^{(0)}, \partial_{j} \tilde{\Lambda}\right]\right] \\
& +4 \Pi^{i j}\left[\partial_{i} \Phi^{(0)}, \partial_{j} \square^{-1} \eta^{k \alpha}\left[\partial_{\alpha} \Phi^{(0)}, \partial_{k} \tilde{\Lambda}\right]\right] \\
= & i \square\left[\tilde{\Lambda}, \Phi^{(1)}\right]-2 i \eta^{i \alpha}\left[\partial_{i} \tilde{\Lambda}, \partial_{\alpha} \Phi^{(1)}\right]-i\left[\tilde{\Lambda}, \square \Phi^{(1)}\right]+\Pi^{i j}\left[\tilde{\Lambda},\left[\partial_{i} \Phi^{(0)}, \partial_{j} \Phi^{(0)}\right]\right] \\
& -2 \Pi^{i j}\left[\partial_{i} \Phi^{(0)},\left[\Phi^{(0)}, \partial_{j} \tilde{\Lambda}\right]\right]+4 \Pi^{i j}\left[\partial_{i} \Phi^{(0)}, \partial_{j} \square^{-1} \eta^{k \alpha}\left[\partial_{\alpha} \Phi^{(0)}, \partial_{k} \tilde{\Lambda}\right]\right]
\end{aligned}
$$

where we used the Jacobi identity to get to the third line and $\square \tilde{\Lambda}=0$ to get to the fourth line. Then, using the eom for $\Phi^{(1)}$, we are left with

$$
\begin{aligned}
\delta_{\tilde{\Lambda}} \Phi^{(2)}= & -i\left[\Phi^{(1)}, \tilde{\Lambda}\right]+2 i \square^{-1} \eta^{i \alpha}\left[\partial_{\alpha} \Phi^{(1)}, \partial_{i} \tilde{\Lambda}\right] \\
& -2 \square^{-1} \Pi^{i j}\left[\partial_{i} \Phi^{(0)},\left[\Phi^{(0)}, \partial_{j} \tilde{\Lambda}\right]\right]+4 \square^{-1} \Pi^{i j}\left[\partial_{i} \Phi^{(0)}, \partial_{j} \square^{-1} \eta^{k \alpha}\left[\partial_{\alpha} \Phi^{(0)}, \partial_{k} \tilde{\Lambda}\right]\right]
\end{aligned}
$$

Next, we look at the gravity transformation. Our seeds are (5.38):

$$
\begin{aligned}
\delta_{\tilde{\lambda}} \phi^{(0)} & =\frac{1}{3} \Omega_{i}{ }^{\alpha} x^{i} \partial_{\alpha} \tilde{\lambda} \\
\delta_{\tilde{\lambda}} \phi^{(1)} & =\frac{1}{2}\left\{\phi^{(0)}, \tilde{\lambda}\right\}-\square^{-1} \eta^{i \alpha}\left\{\partial_{\alpha} \phi^{(0)}, \partial_{i} \tilde{\lambda}\right\}
\end{aligned}
$$

We will now use the e.o.m. at second order in $\phi$,

$$
\square \phi^{(2)}=\Pi^{i j} \Pi^{k l} \partial_{i} \partial_{k} \phi^{(0)} \partial_{j} \partial_{l} \phi^{(1)}
$$

to derive $\delta_{\tilde{\lambda}} \phi^{(2)}$. We have

$$
\begin{aligned}
\square \delta_{\tilde{\lambda}} \phi^{(2)}= & \frac{1}{2} \Pi^{i j} \Pi^{k l} \partial_{i} \partial_{k} \delta_{\tilde{\lambda}} \phi^{(0)} \partial_{j} \partial_{l} \phi^{(1)}+\frac{1}{2} \Pi^{i j} \Pi^{k l} \partial_{i} \partial_{k} \phi^{(0)} \partial_{j} \partial_{l} \delta_{\tilde{\lambda}} \phi^{(1)} \\
= & \Pi^{i j} \Pi^{k l} \partial_{i}\left(\Omega_{k}^{\alpha} \partial_{\alpha} \tilde{\lambda}\right) \partial_{j} \partial_{l} \phi^{(1)}+\frac{1}{2} \Pi^{i j} \Pi^{k l} \partial_{i} \partial_{k} \phi^{(0)} \partial_{j}\left\{\partial_{l} \phi^{(0)}, \tilde{\lambda}\right\} \\
& +\frac{1}{2} \Pi^{i j} \Pi^{k l} \partial_{i} \partial_{k} \phi^{(0)} \partial_{j}\left\{\phi^{(0)}, \partial_{l} \tilde{\lambda}\right\}-\Pi^{i j} \Pi^{k l} \partial_{i} \partial_{k} \phi^{(0)} \partial_{j} \partial_{l} \square^{-1} \eta^{i \alpha}\left\{\partial_{\alpha} \phi^{(0)}, \partial_{i} \tilde{\lambda}\right\} \\
= & -\eta^{l \alpha} \Pi^{i j} \partial_{i} \partial_{\alpha} \tilde{\lambda} \partial_{j} \partial_{l} \phi^{(1)}-\frac{1}{4} \Pi^{k l}\left\{\tilde{\lambda},\left\{\partial_{k} \phi^{(0)}, \partial_{l} \phi^{(0)}\right\}\right\} \\
& +\frac{1}{2} \Pi^{i j} \Pi^{k l} \partial_{i} \partial_{k} \phi^{(0)} \partial_{j}\left\{\phi^{(0)}, \partial_{l} \tilde{\lambda}\right\}-\Pi^{i j} \Pi^{k l} \partial_{i} \partial_{k} \phi^{(0)} \partial_{j} \partial_{l} \square^{-1} \eta^{i \alpha}\left\{\partial_{\alpha} \phi^{(0)}, \partial_{i} \tilde{\lambda}\right\} \\
= & -\frac{1}{2} \square\left\{\tilde{\lambda}, \phi^{(1)}\right\}+\eta^{l \alpha}\left\{\partial_{l} \tilde{\lambda}, \partial_{\alpha} \phi^{(1)}\right\}+\frac{1}{2}\left\{\tilde{\lambda}, \square \phi^{(1)}\right\}-\frac{1}{4} \Pi^{k l}\left\{\tilde{\lambda},\left\{\partial_{k} \phi^{(0)}, \partial_{l} \phi^{(0)}\right\}\right\} \\
& +\frac{1}{2} \Pi^{i j} \Pi^{k l} \partial_{i} \partial_{k} \phi^{(0)} \partial_{j}\left\{\phi^{(0)}, \partial_{l} \tilde{\lambda}\right\}-\Pi^{i j} \Pi^{k l} \partial_{i} \partial_{k} \phi^{(0)} \partial_{j} \partial_{l} \square^{-1} \eta^{i \alpha}\left\{\partial_{\alpha} \phi^{(0)}, \partial_{i} \tilde{\lambda}\right\}
\end{aligned}
$$

where we used the Jacobi identity for the Poisson bracket to get to the third line and $\square \tilde{\lambda}=0$ to get to the fourth line. Then, using the e.o.m. for $\phi^{(1)}$, we get

$$
\begin{aligned}
\delta_{\tilde{\lambda}} \phi^{(2)}= & \frac{1}{2}\left\{\phi^{(1)}, \tilde{\lambda}\right\}-\square^{-1} \eta^{i \alpha}\left\{\partial_{\alpha} \phi^{(1)}, \partial_{i} \tilde{\lambda}\right\} \\
& +\frac{1}{2} \square^{-1} \Pi^{i j}\left\{\partial_{i} \phi^{(0)},\left\{\phi^{(0)}, \partial_{j} \tilde{\lambda}\right\}\right\}-\square^{-1} \Pi^{i j}\left\{\partial_{i} \phi^{(0)}, \partial_{j} \square^{-1} \eta^{i \alpha}\left\{\partial_{\alpha} \phi^{(0)}, \partial_{i} \tilde{\lambda}\right\}\right\}
\end{aligned}
$$


and comparing with the YM transformation (B.14) we see that it double copies correctly under the rules (B.1).

\section{B.2 Recursive construction at arbitrary orders}

We will demonstrate recursively that the double copy rules (B.1) work at all orders in perturbation theory. The proof applies simultaneously to the first and second family of symmetries (and, indeed, to the infinite set of families constructed in section 5.5). Start by assuming we have shown that the transformation rules for self-dual YM up to some order $(n-1)$ :

$$
\delta_{\Lambda} \Phi^{(0)}, \delta_{\Lambda} \Phi^{(1)}, \cdots, \delta_{\Lambda} \Phi^{(n-1)}
$$

double copy correctly, under the rules (B.1), into the respective gravity transformations:

$$
\delta_{\lambda} \phi^{(0)}, \delta_{\lambda} \phi^{(1)}, \cdots, \delta_{\lambda} \phi^{(n-1)}
$$

We now treat the transformations $\delta_{\tilde{\Lambda}} \Phi^{(0)}, \cdots, \delta_{\tilde{\Lambda}} \Phi^{(n-1)}$ as seeds, and make use of the YM self-dual equation at order $n$ :

$$
\square \Phi^{(n)}=-i \Pi^{i j} \sum_{m+p=n-1}\left[\partial_{i} \Phi^{(m)}, \partial_{j} \Phi^{(p)}\right]
$$

to derive

$$
\delta_{\tilde{\Lambda}} \Phi^{(n)}=-i \Pi^{i j} \square^{-1} \sum_{m+p=n-1}\left[\partial_{i} \delta_{\tilde{\Lambda}} \Phi^{(m)}, \partial_{j} \Phi^{(p)}\right]-i \Pi^{i j} \square^{-1} \sum_{m+p=n-1}\left[\partial_{i} \Phi^{(m)}, \partial_{j} \delta_{\tilde{\Lambda}} \Phi^{(p)}\right]
$$

Then using the d.c. rules (B.1) and the assumption that the double copy works up to order $(n-1)$ we get

$$
\delta_{\tilde{\lambda}} \phi^{(n)}=\frac{1}{2} \Pi^{i j} \square^{-1} \sum_{m+p=n-1}\left\{\partial_{i} \delta_{\tilde{\lambda}} \phi^{(m)}, \partial_{j} \phi^{(p)}\right\}+\frac{1}{2} \Pi^{i j} \square^{-1} \sum_{m+p=n-1}\left\{\partial_{i} \phi^{(m)}, \partial_{j} \delta_{\tilde{\lambda}} \phi^{(p)}\right\}
$$

Separately, we can directly derive the gravity transformation by taking $\delta_{\tilde{\lambda}} \phi^{(0)}, \cdots, \delta_{\tilde{\lambda}} \phi^{(n-1)}$ as seeds, and using the gravity self-dual equation at order $\mathrm{n}$ :

$$
\square \phi^{(n)}=\frac{1}{2} \Pi^{i j} \sum_{m+p=n-1}\left\{\partial_{i} \phi^{(m)}, \partial_{j} \phi^{(p)}\right\}
$$

we get

$$
\square \delta_{\tilde{\lambda}} \phi^{(n)}=\frac{1}{2} \Pi^{i j} \sum_{m+p=n-1}\left\{\partial_{i} \delta_{\tilde{\lambda}} \phi^{(m)}, \partial_{j} \phi^{(p)}\right\}+\frac{1}{2} \Pi^{i j} \sum_{m+p=n-1}\left\{\partial_{i} \phi^{(m)}, \partial_{j} \delta_{\tilde{\lambda}} \phi^{(p)}\right\}
$$

so indeed

$$
\delta_{\tilde{\lambda}} \phi^{(n)}=\frac{1}{2} \Pi^{i j} \square^{-1} \sum_{m+p=n-1}\left\{\partial_{i} \delta_{\tilde{\lambda}} \phi^{(m)}, \partial_{j} \phi^{(p)}\right\}+\frac{1}{2} \Pi^{i j} \square^{-1} \sum_{m+p=n-1}\left\{\partial_{i} \phi^{(m)}, \partial_{j} \delta_{\tilde{\lambda}} \phi^{(p)}\right\}
$$

as needed. As seen in this section, the fact that the DC rules continue to hold at all orders can be traced back to the fact that the e.o.m. themselves exhibit a DC structure. 


\section{Comparison with convolution DC}

At zeroth order in the fields there exists an alternative DC construction for gravity and YM symmetries, based on a convolution dictionary [43-45]. In this appendix we will show that our DC is consistent with the convolution one where they overlap. Since the latter relates linearized YM and gravity gauge symmetries, we will compare it with our first family at zeroth order in the fields.

For simplicity, we present the comparison in the case of $(2,2)$ signature, ${ }^{17}$ where $Z$ and $\bar{Z}$ are treated as independent real variables. This will allow us to perform Fourier transforms along $Z$ and $\bar{Z}$ independently.

In momentum space, the convolution DC relates a linearized gravity diffeomorphism $\tilde{\xi}_{\mu}(p)$ to a linearized YM gauge parameter $\tilde{\Lambda}(p)$ by ${ }^{18}$

$$
\tilde{\xi}_{\mu}(p)=\operatorname{Tr}\left[\tilde{\Lambda}(p) \tilde{\varphi}^{-1}(p) \tilde{\mathcal{A}}_{\mu}(p)\right]
$$

where $\varphi$ is the biadjoint "spectactor" scalar field. Since $A_{i}=0$, we immediately see that

$$
\xi_{i}=0
$$

consistent with (3.20). Next we note that since $\Lambda=\Lambda(y)$ is independent of $x^{i}=(U, \bar{Z})$ it follows that

$$
\tilde{\Lambda}(p)=\delta\left(p_{U}\right) \delta\left(p_{\bar{Z}}\right) \tilde{\Lambda}\left(p_{V}, p_{Z}\right) .
$$

Substituting in (C.1), we find that $\tilde{\xi}_{\mu}$ is proportional to $\delta\left(p_{U}\right) \delta\left(p_{\bar{Z}}\right)$. Hence, when transformed back to position space, it can only depend on the $y$ variables

$$
\xi_{\alpha}=\xi_{\alpha}(y)
$$

consistent with (3.20).

Open Access. This article is distributed under the terms of the Creative Commons Attribution License (CC-BY 4.0), which permits any use, distribution and reproduction in any medium, provided the original author(s) and source are credited.

\section{References}

[1] Z. Bern, J.J. Carrasco, M. Chiodaroli, H. Johansson and R. Roiban, The Duality Between Color and Kinematics and its Applications, arXiv:1909.01358 [INSPIRE].

[2] L. Borsten, Gravity as the square of gauge theory: a review, Riv. Nuovo Cim. 43 (2020) 97.

[3] L. Borsten and M.J. Duff, Gravity as the square of Yang-Mills?, Phys. Scripta 90 (2015) 108012 [arXiv: 1602 .08267] [INSPIRE].

[4] Z. Bern, J.J.M. Carrasco and H. Johansson, New Relations for Gauge-Theory Amplitudes, Phys. Rev. D 78 (2008) 085011 [arXiv:0805.3993] [INSPIRE].

\footnotetext{
${ }^{17}$ See e.g. [125] for a recent discussion.

${ }^{18}$ Note $\tilde{\Lambda}$ is the Fourier transform of the gauge parameter $\Lambda$. It should not be confused with the parameter of the second family of symmetries, which in the main text is also denoted by $\tilde{\Lambda}$. For simplicity we have assumed that the two gauge sectors are identical.
} 
[5] Z. Bern, J.J.M. Carrasco and H. Johansson, Perturbative Quantum Gravity as a Double Copy of Gauge Theory, Phys. Rev. Lett. 105 (2010) 061602 [arXiv: 1004.0476] [INSPIRE].

[6] Z. Bern, T. Dennen, Y.-t. Huang and M. Kiermaier, Gravity as the Square of Gauge Theory, Phys. Rev. D 82 (2010) 065003 [arXiv:1004.0693] [InSPIRE].

[7] Z. Bern, J.J.M. Carrasco, W.-M. Chen, H. Johansson, R. Roiban and M. Zeng, Five-loop four-point integrand of $N=8$ supergravity as a generalized double copy, Phys. Rev. D 96 (2017) 126012 [arXiv:1708.06807] [INSPIRE].

[8] Z. Bern, S. Davies, T. Dennen and Y.-t. Huang, Absence of Three-Loop Four-Point Divergences in $N=4$ Supergravity, Phys. Rev. Lett. 108 (2012) 201301 [arXiv:1202.3423] [INSPIRE].

[9] Z. Bern et al., Ultraviolet Properties of $\mathcal{N}=8$ Supergravity at Five Loops, Phys. Rev. D 98 (2018) 086021 [arXiv: 1804.09311] [INSPIRE].

[10] N.E.J. Bjerrum-Bohr, P.H. Damgaard, R. Monteiro and D. O'Connell, Algebras for Amplitudes, JHEP 06 (2012) 061 [arXiv:1203.0944] [INSPIRE].

[11] R. Monteiro and D. O'Connell, The Kinematic Algebras from the Scattering Equations, JHEP 03 (2014) 110 [arXiv:1311.1151] [INSPIRE].

[12] A. Luna et al., Perturbative spacetimes from Yang-Mills theory, JHEP 04 (2017) 069 [arXiv: 1611.07508] [INSPIRE].

[13] W.D. Goldberger and A.K. Ridgway, Radiation and the classical double copy for color charges, Phys. Rev. D 95 (2017) 125010 [arXiv:1611.03493] [InSPIRE].

[14] G.L. Cardoso, S. Nagy and S. Nampuri, A double copy for $\mathcal{N}=2$ supergravity: a linearised tale told on-shell, JHEP 10 (2016) 127 [arXiv: 1609.05022] [INSPIRE].

[15] G. Cardoso, S. Nagy and S. Nampuri, Multi-centered $\mathcal{N}=2$ BPS black holes: a double copy description, JHEP 04 (2017) 037 [arXiv:1611.04409] [INSPIRE].

[16] A. Luna, S. Nagy and C. White, The convolutional double copy: a case study with a point, JHEP 09 (2020) 062 [arXiv: 2004.11254] [INSPIRE].

[17] W.D. Goldberger, J. Li and S.G. Prabhu, Spinning particles, axion radiation, and the classical double copy, Phys. Rev. D 97 (2018) 105018 [arXiv:1712.09250] [InSPIRE].

[18] R. Monteiro, D. O'Connell, D.P. Veiga and M. Sergola, Classical Solutions and their Double Copy in Split Signature, arXiv:2012.11190 [INSPIRE].

[19] R. Monteiro, D. O'Connell and C.D. White, Black holes and the double copy, JHEP 12 (2014) 056 [arXiv: 1410.0239] [INSPIRE].

[20] A.K. Ridgway and M.B. Wise, Static Spherically Symmetric Kerr-Schild Metrics and Implications for the Classical Double Copy, Phys. Rev. D 94 (2016) 044023 [arXiv: 1512.02243] [INSPIRE].

[21] C.D. White, Exact solutions for the biadjoint scalar field, Phys. Lett. B 763 (2016) 365 [arXiv: 1606.04724] [INSPIRE].

[22] A. Luna, R. Monteiro, I. Nicholson and D. O'Connell, Type D Spacetimes and the Weyl Double Copy, Class. Quant. Grav. 36 (2019) 065003 [arXiv:1810.08183] [INSPIRE].

[23] D.S. Berman, E. Chacón, A. Luna and C.D. White, The self-dual classical double copy, and the Eguchi-Hanson instanton, JHEP 01 (2019) 107 [arXiv:1809.04063] [INSPIRE].

[24] I. Bah, R. Dempsey and P. Weck, Kerr-Schild Double Copy and Complex Worldlines, JHEP 02 (2020) 180 [arXiv: 1910. 04197] [INSPIRE]. 
[25] L. Alfonsi, C.D. White and S. Wikeley, Topology and Wilson lines: global aspects of the double copy, JHEP 07 (2020) 091 [arXiv: 2004.07181] [INSPIRE].

[26] K. Kim, K. Lee, R. Monteiro, I. Nicholson and D. Peinador Veiga, The Classical Double Copy of a Point Charge, JHEP 02 (2020) 046 [arXiv: 1912.02177] [INSPIRE].

[27] N. Bahjat-Abbas, R. Stark-Muchão and C.D. White, Monopoles, shockwaves and the classical double copy, JHEP 04 (2020) 102 [arXiv: 2001.09918] [INSPIRE].

[28] E. Lescano and J.A. Rodríguez, $\mathcal{N}=1$ supersymmetric Double Field Theory and the generalized Kerr-Schild ansatz, JHEP 10 (2020) 148 [arXiv: 2002.07751] [INSPIRE].

[29] P.-J. De Smet and C.D. White, Extended solutions for the biadjoint scalar field, Phys. Lett. $B \mathbf{7 7 5}$ (2017) 163 [arXiv:1708.01103] [INSPIRE].

[30] K. Lee, Kerr-Schild Double Field Theory and Classical Double Copy, JHEP 10 (2018) 027 [arXiv: 1807.08443] [INSPIRE].

[31] C. Keeler, T. Manton and N. Monga, From Navier-Stokes to Maxwell via Einstein, JHEP 08 (2020) 147 [arXiv: 2005. 04242] [inSPIRE].

[32] R. Alawadhi, D.S. Berman and B. Spence, Weyl doubling, JHEP 09 (2020) 127 [arXiv: 2007.03264] [INSPIRE].

[33] H. Godazgar, M. Godazgar, R. Monteiro, D.P. Veiga and C.N. Pope, Weyl Double Copy for Gravitational Waves, Phys. Rev. Lett. 126 (2021) 101103 [arXiv:2010.02925] [INSPIRE].

[34] C.D. White, Twistorial Foundation for the Classical Double Copy, Phys. Rev. Lett. 126 (2021) 061602 [arXiv: 2012.02479] [inSPIRE].

[35] D.S. Berman, K. Kim and K. Lee, The Classical Double Copy for M-theory from a Kerr-Schild Ansatz for Exceptional Field Theory, arXiv:2010.08255 [INSPIRE].

[36] J.J.M. Carrasco, C.R. Mafra and O. Schlotterer, Semi-abelian Z-theory: NLSM+ $\phi^{3}$ from the open string, JHEP 08 (2017) 135 [arXiv:1612.06446] [INSPIRE].

[37] J.J.M. Carrasco, C.R. Mafra and O. Schlotterer, Abelian Z-theory: NLSM amplitudes and $\alpha^{\prime}$-corrections from the open string, JHEP 06 (2017) 093 [arXiv:1608.02569] [INSPIRE].

[38] C. Cheung and C.-H. Shen, Symmetry for Flavor-Kinematics Duality from an Action, Phys. Rev. Lett. 118 (2017) 121601 [arXiv:1612.00868] [INSPIRE].

[39] L. Borsten, $D=6, \mathcal{N}=(2,0)$ and $\mathcal{N}=(4,0)$ theories, Phys. Rev. D 97 (2018) 066014 [arXiv: 1708.02573] [INSPIRE].

[40] L. Borsten, I. Jubb, V. Makwana and S. Nagy, Gauge $\times$ gauge on spheres, JHEP 06 (2020) 096 [arXiv: 1911.12324] [INSPIRE].

[41] S. Nagy, Chiral Squaring, JHEP 07 (2016) 142 [arXiv:1412.4750] [inSPIRE].

[42] F. Cachazo, S. He and E.Y. Yuan, Scattering Equations and Matrices: From Einstein To Yang-Mills, DBI and NLSM, JHEP 07 (2015) 149 [arXiv:1412.3479] [INSPIRE].

[43] A. Anastasiou, L. Borsten, M.J. Duff, L.J. Hughes and S. Nagy, Yang-Mills origin of gravitational symmetries, Phys. Rev. Lett. 113 (2014) 231606 [arXiv:1408.4434] [INSPIRE].

[44] A. Anastasiou, L. Borsten, M.J. Duff, S. Nagy and M. Zoccali, Gravity as Gauge Theory Squared: A Ghost Story, Phys. Rev. Lett. 121 (2018) 211601 [arXiv:1807. 02486] [INSPIRE].

[45] G. Lopes Cardoso, G. Inverso, S. Nagy and S. Nampuri, Comments on the double copy construction for gravitational theories, in 17th Hellenic School and Workshops on Elementary Particle Physics and Gravity PoS CORFU2017 (2018) 177 [arXiv: 1803.07670] [INSPIRE] [http://inspirehep.net/record/1663475/files/1803.07670.pdf]. 
[46] R. Alawadhi, D.S. Berman, B. Spence and D. Peinador Veiga, S-duality and the double copy, JHEP 03 (2020) 059 [arXiv: 1911.06797] [INSPIRE].

[47] A. Banerjee, E.O. Colgáin, J.A. Rosabal and H. Yavartanoo, Ehlers as EM duality in the double copy, Phys. Rev. D 102 (2020) 126017 [arXiv:1912.02597] [InSPIRE].

[48] L. Borsten, I. Jubb, V. Makwana and S. Nagy, Gauge $\times$ Gauge $=$ Gravity on Homogeneous Spaces using Tensor Convolutions, to appear.

[49] A. Anastasiou, L. Borsten, M.J. Duff, A. Marrani, S. Nagy and M. Zoccali, Are all supergravity theories Yang-Mills squared?, Nucl. Phys. B 934 (2018) 606 [arXiv: 1707.03234] [INSPIRE].

[50] L. Borsten, M.J. Duff, L.J. Hughes and S. Nagy, Magic Square from Yang-Mills Squared, Phys. Rev. Lett. 112 (2014) 131601 [arXiv:1301.4176] [INSPIRE].

[51] A. Anastasiou, L. Borsten, M.J. Duff, L.J. Hughes and S. Nagy, A magic pyramid of supergravities, JHEP 04 (2014) 178 [arXiv:1312.6523] [INSPIRE].

[52] A. Anastasiou, L. Borsten, M.J. Hughes and S. Nagy, Global symmetries of Yang-Mills squared in various dimensions, JHEP 01 (2016) 148 [arXiv:1502.05359] [INSPIRE].

[53] A. Anastasiou, L. Borsten, M.J. Duff, A. Marrani, S. Nagy and M. Zoccali, The Mile High Magic Pyramid, Contemp. Math. 721 (2019) 1 [arXiv:1711.08476] [INSPIRE].

[54] M. Tolotti and S. Weinzierl, Construction of an effective Yang-Mills Lagrangian with manifest BCJ duality, JHEP 07 (2013) 111 [arXiv:1306.2975] [INSPIRE].

[55] J. Plefka, J. Steinhoff and W. Wormsbecher, Effective action of dilaton gravity as the classical double copy of Yang-Mills theory, Phys. Rev. D 99 (2019) 024021 [arXiv: 1807. 09859] [INSPIRE].

[56] J. Plefka, C. Shi and T. Wang, Double copy of massive scalar QCD, Phys. Rev. D 101 (2020) 066004 [arXiv:1911.06785] [inSPIRE].

[57] L. Borsten, B. Jurčo, H. Kim, T. Macrelli, C. Sämann and M. Wolf, BRST-Lagrangian Double Copy of Yang-Mills Theory, arXiv:2007.13803 [INSPIRE].

[58] L. Borsten and S. Nagy, The pure BRST Einstein-Hilbert Lagrangian from the double-copy to cubic order, JHEP 07 (2020) 093 [arXiv: 2004.14945] [INSPIRE].

[59] P. Ferrero and D. Francia, On the Lagrangian formulation of the double copy to cubic order, JHEP 02 (2021) 213 [arXiv:2012.00713] [InSPIRE].

[60] R. Penrose, Asymptotic properties of fields and space-times, Phys. Rev. Lett. 10 (1963) 66 [INSPIRE].

[61] H. Bondi, M.G.J. Van der Burg and A.W.K Metzner, Gravitational waves in general relativity, VII. Waves from axisymmetric isolated systems, Proc. Roy. Soc. Lond. A 269 (1962) 21.

[62] R.K. Sachs, Gravitational waves in general relativity, VIII. Waves in asymptotically flat space-times, Proc. Roy. Soc. Lond. A 270 (1962) 103.

[63] A. Ashtekar, Radiative Degrees of Freedom of the Gravitational Field in Exact General Relativity, J. Math. Phys. 22 (1981) 2885 [INSPIRE].

[64] A. Ashtekar and M. Streubel, Symplectic Geometry of Radiative Modes and Conserved Quantities at Null Infinity, Proc. Roy. Soc. Lond. A 376 (1981) 585.

[65] A. Ashtekar, Asymptotic quantization: based on 1984 Naples lectures, Bibliopolis Naples, Italy (1987). 
[66] G. Barnich and C. Troessaert, Symmetries of asymptotically flat 4 dimensional spacetimes at null infinity revisited, Phys. Rev. Lett. 105 (2010) 111103 [arXiv:0909.2617] [InSPIRE].

[67] A. Strominger, On BMS Invariance of Gravitational Scattering, JHEP 07 (2014) 152 [arXiv: 1312.2229] [INSPIRE].

[68] A. Strominger, Asymptotic Symmetries of Yang-Mills Theory, JHEP 07 (2014) 151 [arXiv: 1308.0589] [INSPIRE].

[69] T. He, V. Lysov, P. Mitra and A. Strominger, BMS supertranslations and Weinberg's soft graviton theorem, JHEP 05 (2015) 151 [arXiv:1401.7026] [INSPIRE].

[70] A. Strominger, Lectures on the Infrared Structure of Gravity and Gauge Theory, arXiv: 1703.05448 [INSPIRE].

[71] A. Ashtekar, M. Campiglia and A. Laddha, Null infinity, the BMS group and infrared issues, Gen. Rel. Grav. 50 (2018) 140 [arXiv:1808.07093] [INSPIRE].

[72] S. Pasterski, Implications of Superrotations, Phys. Rept. 829 (2019) 1 [arXiv:1905.10052] [INSPIRE].

[73] N.E.J. Bjerrum-Bohr, P.H. Damgaard, T. Sondergaard and P. Vanhove, The Momentum Kernel of Gauge and Gravity Theories, JHEP 01 (2011) 001 [arXiv:1010.3933] [INSPIRE].

[74] S. Oxburgh and C.D. White, BCJ duality and the double copy in the soft limit, JHEP 02 (2013) 127 [arXiv: 1210.1110] [INSPIRE].

[75] S.G. Naculich, H. Nastase and H.J. Schnitzer, All-loop infrared-divergent behavior of most-subleading-color gauge-theory amplitudes, JHEP 04 (2013) 114 [arXiv:1301.2234] [INSPIRE].

[76] S. He, Y.-t. Huang and C. Wen, Loop Corrections to Soft Theorems in Gauge Theories and Gravity, JHEP 12 (2014) 115 [arXiv:1405.1410] [INSPIRE].

[77] C.D. White, Diagrammatic insights into next-to-soft corrections, Phys. Lett. B 737 (2014) 216 [arXiv:1406.7184] [INSPIRE].

[78] A. Sabio Vera and M.A. Vazquez-Mozo, The Double Copy Structure of Soft Gravitons, JHEP 03 (2015) 070 [arXiv: 1412.3699] [InSPIRE].

[79] A. Luna, S. Melville, S.G. Naculich and C.D. White, Next-to-soft corrections to high energy scattering in QCD and gravity, JHEP 01 (2017) 052 [arXiv:1611.02172] [INSPIRE].

[80] A.P.V. and A. Manu, Classical double copy from Color Kinematics duality: A proof in the soft limit, Phys. Rev. D 101 (2020) 046014 [arXiv:1907.10021] [INSPIRE].

[81] E. Casali and A. Puhm, Double Copy for Celestial Amplitudes, Phys. Rev. Lett. 126 (2021) 101602 [arXiv:2007.15027] [INSPIRE].

[82] E. Casali and A. Sharma, Celestial double copy from the worldsheet, arXiv:2011.10052 [INSPIRE].

[83] N. Kalyanapuram, Soft S-Matrices, Defects and the Double Copy on the Celestial Sphere, arXiv:2011.11412 [INSPIRE].

[84] N. Kalyanapuram, Gauge and Gravity Amplitudes on the Celestial Sphere, arXiv: 2012.04579 [INSPIRE].

[85] Y.-T. Huang, U. Kol and D. O'Connell, Double copy of electric-magnetic duality, Phys. Rev. D 102 (2020) 046005 [arXiv: 1911.06318] [INSPIRE].

[86] W.T. Emond, Y.-T. Huang, U. Kol, N. Moynihan and D. O'Connell, Amplitudes from Coulomb to Kerr-Taub-NUT, arXiv:2010.07861 [INSPIRE]. 
[87] R. Monteiro and D. O'Connell, The Kinematic Algebra From the Self-Dual Sector, JHEP 07 (2011) 007 [arXiv: 1105.2565] [INSPIRE].

[88] C.-H. Fu and K. Krasnov, Colour-Kinematics duality and the Drinfeld double of the Lie algebra of diffeomorphisms, JHEP 01 (2017) 075 [arXiv: 1603.02033] [INSPIRE].

[89] G. Chen, H. Johansson, F. Teng and T. Wang, On the kinematic algebra for BCJ numerators beyond the MHV sector, JHEP 11 (2019) 055 [arXiv:1906.10683] [INSPIRE].

[90] G. Elor, K. Farnsworth, M.L. Graesser and G. Herczeg, The Newman-Penrose Map and the Classical Double Copy, JHEP 12 (2020) 121 [arXiv:2006.08630] [INSPIRE].

[91] E. Chacón, H. García-Compeán, A. Luna, R. Monteiro and C.D. White, New heavenly double copies, arXiv:2008.09603 [INSPIRE].

[92] R.H. Boels, R.S. Isermann, R. Monteiro and D. O'Connell, Colour-Kinematics Duality for One-Loop Rational Amplitudes, JHEP 04 (2013) 107 [arXiv:1301.4165] [INSPIRE].

[93] J.F. Plebanski, Some solutions of complex Einstein equations, J. Math. Phys. 16 (1975) 2395 [INSPIRE].

[94] M.K. Prasad, A. Sinha and L.-L. Wang, Nonlocal Continuity Equations for Selfdual SU(N) Yang-Mills Fields, Phys. Lett. B 87 (1979) 237 [INSPIRE].

[95] L. Dolan, Kac-Moody Algebras and Exact Solvability in Hadronic Physics, Phys. Rept. 109 (1984) 1 [INSPIRE].

[96] A. Parkes, A Cubic action for selfdual Yang-Mills, Phys. Lett. B 286 (1992) 265 [hep-th/9203074] [INSPIRE].

[97] G. Chalmers and W. Siegel, The Selfdual sector of QCD amplitudes, Phys. Rev. D 54 (1996) 7628 [hep-th/9606061] [INSPIRE].

[98] D. Cangemi, Selfduality and maximally helicity violating QCD amplitudes, Int. J. Mod. Phys. A 12 (1997) 1215 [hep-th/9610021] [INSPIRE].

[99] A.D. Popov, M. Bordemann and H. Romer, Symmetries, currents and conservation laws of selfdual gravity, Phys. Lett. B 385 (1996) 63 [hep-th/9606077] [INSPIRE].

[100] A.D. Popov, Selfdual Yang-Mills: Symmetries and moduli space, Rev. Math. Phys. 11 (1999) 1091 [hep-th/9803183] [INSPIRE].

[101] W.A. Bardeen, Selfdual Yang-Mills theory, integrability and multiparton amplitudes, Prog. Theor. Phys. Suppl. 123 (1996) 1 [inSPIRE].

[102] Q.-H. Park, Selfdual Gravity as a Large N Limit of the Two-dimensional Nonlinear $\sigma$ Model, Phys. Lett. B 238 (1990) 287 [INSPIRE].

[103] V. Husain, Selfdual gravity as a two-dimensional theory and conservation laws, Class. Quant. Grav. 11 (1994) 927 [gr-qc/9310003] [INSPIRE].

[104] V. Husain, The Affine symmetry of selfdual gravity, J. Math. Phys. 36 (1995) 6897 [hep-th/9410072] [INSPIRE].

[105] M. Wolf, On hidden symmetries of a super gauge theory and twistor string theory, JHEP 02 (2005) 018 [hep-th/0412163] [INSPIRE].

[106] A.D. Popov and M. Wolf, Hidden symmetries and integrable hierarchy of the $N=4$ supersymmetric Yang-Mills equations, Commun. Math. Phys. 275 (2007) 685 [hep-th/0608225] [INSPIRE].

[107] D. Kapec and P. Mitra, A d-Dimensional Stress Tensor for Mink ${ }_{d+2}$ Gravity, JHEP 05 (2018) 186 [arXiv:1711.04371] [InSPIRE]. 
[108] T. He, P. Mitra and A. Strominger, 2D Kac-Moody Symmetry of 4 D Yang-Mills Theory, JHEP 10 (2016) 137 [arXiv: 1503. 02663] [INSPIRE].

[109] M. Campiglia and A. Laddha, Subleading soft photons and large gauge transformations, JHEP 11 (2016) 012 [arXiv:1605.09677] [INSPIRE].

[110] A. Laddha and P. Mitra, Asymptotic Symmetries and Subleading Soft Photon Theorem in Effective Field Theories, JHEP 05 (2018) 132 [arXiv:1709.03850] [INSPIRE].

[111] T. He and P. Mitra, Asymptotic symmetries in $(d+2)$-dimensional gauge theories, JHEP 10 (2019) 277 [arXiv: 1903.03607] [INSPIRE].

[112] M. Campiglia and J. Peraza, Charge algebra for large gauge symmetries at $O(r)$, to appear.

[113] M. Campiglia and A. Laddha, New symmetries for the Gravitational S-matrix, JHEP 04 (2015) 076 [arXiv: 1502.02318] [INSPIRE].

[114] G. Compère, A. Fiorucci and R. Ruzziconi, Superboost transitions, refraction memory and super-Lorentz charge algebra, JHEP 11 (2018) 200 [Erratum ibid. 04 (2020) 172] [arXiv: 1810.00377] [INSPIRE].

[115] M. Campiglia and J. Peraza, Generalized BMS charge algebra, Phys. Rev. D 101 (2020) 104039 [arXiv: 2002.06691] [INSPIRE].

[116] S. Ananth, L. Brink and S. Majumdar, BMS algebra from residual gauge invariance in light-cone gravity, arXiv:2101.00019 [INSPIRE].

[117] A. Ashtekar and M. Varadarajan, Gravitational Dynamics - A Novel Shift in the Hamiltonian Paradigm, Universe 7 (2021) 13 [arXiv: 2012.12094] [INSPIRE].

[118] A.A. Rosly and K.G. Selivanov, On amplitudes in selfdual sector of Yang-Mills theory, Phys. Lett. B 399 (1997) 135 [hep-th/9611101] [INSPIRE].

[119] L.J. Mason and D. Skinner, Gravity, Twistors and the MHV Formalism, Commun. Math. Phys. 294 (2010) 827 [arXiv: 0808. 3907] [INSPIRE].

[120] M. Campiglia and A. Laddha, Sub-subleading soft gravitons and large diffeomorphisms, JHEP 01 (2017) 036 [arXiv:1608.00685] [INSPIRE].

[121] M. Campiglia and A. Laddha, Asymptotic charges in massless QED revisited: A view from Spatial Infinity, JHEP 05 (2019) 207 [arXiv:1810.04619] [INSPIRE].

[122] A. Seraj, Multipole charge conservation and implications on electromagnetic radiation, JHEP 06 (2017) 080 [arXiv:1610.02870] [INSPIRE].

[123] G. Compère, R. Oliveri and A. Seraj, Gravitational multipole moments from Noether charges, JHEP 05 (2018) 054 [arXiv: 1711.08806] [INSPIRE].

[124] G. Compère, Infinite towers of supertranslation and superrotation memories, Phys. Rev. Lett. 123 (2019) 021101 [arXiv: 1904.00280] [INSPIRE].

[125] A. Atanasov, A. Ball, W. Melton, A.-M. Raclariu and A. Strominger, $(2,2)$ Scattering and the Celestial Torus, arXiv:2101.09591 [InSPIRE]. 